\title{
Minireview
}

\section{The synthesis of gemcitabine}

\author{
Kylie Brown ${ }^{\mathrm{a}, \mathrm{b}}$, Michael Dixey ${ }^{\mathrm{a}}$, Alex Weymouth-Wilson ${ }^{\mathrm{b}}$, Bruno Linclau ${ }^{\mathrm{a}, *}$ \\ ${ }^{a}$ Department of Chemistry, University of Southampton, Highfield, Southampton SO17 1BJ, UK \\ ${ }^{\mathrm{b}}$ Dextra Laboratories Ltd, The Science and Technology Centre, Earley Gate, Whiteknights Road, Reading RG6 6BZ, UK
}

\section{A R T I C L E I N F O}

Article history:

Received 30 November 2013

Received in revised form 27 January 2014

Accepted 30 January 2014

Available online 10 February 2014

\section{Keywords:}

Gemcitabine

Fluorinated nucleoside

Fluorosugar

Fluorination

Nucleobase introduction

\begin{abstract}
A B S T R A C T
Gemcitabine is a fluorinated nucleoside currently administered against a number of cancers. It consists of a cytosine base and a 2-deoxy-2,2-difluororibose sugar. The synthetic challenges associated with the introduction of the fluorine atoms, as well as with nucleobase introduction of 2,2-difluorinated sugars, combined with the requirement to have an efficient process suitable for large scale synthesis, have spurred significant activity towards the synthesis of gemcitabine exploring a wide variety of synthetic approaches. In addition, many methods have been developed for selective crystallisation of diastereomeric (including anomeric) mixtures. In that regard, the 2-deoxy-2,2-difluororibose sugar is one of the most investigated fluorinated carbohydrates in terms of its synthesis. The versatility of synthetic methods employed is illustrative of the current state of the art of fluorination methodology for the synthesis of $\mathrm{CF}_{2}$-containing carbohydrates, and involves the use of fluorinated building blocks, as well as nucleophilic and electrophilic fluorination of sugar precursors.
\end{abstract}

(c) 2014 Published by Elsevier Ltd.

\section{Introduction}

Gemcitabine 1 (Fig. 1) is a fluorinated nucleoside analogue. ${ }^{1}$ Originally developed by Lilly, it is an anticancer drug marketed as the $\mathrm{HCl}$ salt under the trade name of Gemzar (Lilly). Whilst the market for gemcitabine continues to grow, the recent expiry of the patent, and consequent availability of generics, have resulted in a decrease in total revenue to below the $\$ 1$ bn dollar mark (Table 1).

In pancreatic cancer, gemcitabine is administered as the sole agent, but in non-small cell lung cancer and bladder cancer, it is given in combination with cisplatin. In ovarian cancer it is given before carboplatin, and in breast cancer after paclitaxel. Gemcitabine is a prodrug; it undergoes intracellular phosphorylation to its active diphosphate and triphosphate form, which inhibits DNA synthesis leading to apoptosis. ${ }^{3,4}$

The clinical success of gemcitabine is somewhat hampered by a short plasma half-life. Gemcitabine. $\mathrm{HCl}$ is only administered via intravenous routes. The dosage of gemcitabine ranges from $1000-1250 \mathrm{mg} / \mathrm{m}^{2}$, dependent upon the type of cancer. ${ }^{3}$ The drug is mainly metabolised by cytidine deaminases, and almost all is excreted in the urine as the corresponding difluorouridine species. New approaches to increase its chemotherapeutic efficiency are under investigation. ${ }^{5}$

\footnotetext{
* Corresponding author. Tel.: +44 2380593816 .

E-mail address: bruno.linclau@soton.ac.uk (B. Linclau).
}

The synthesis of gemcitabine has received much attention. Following the original synthesis by the Eli Lilly team in 1988 featuring a fluorinated building block approach and a nucleobase introduction via displacement of an anomeric mesylate leaving group, many modifications of this synthesis have been reported, mainly towards improving diastereomeric ratios and/or to provide improved methods for separation of the associated diastereomeric mixtures, usually by crystallisation. In addition, alternative syntheses towards the difluororibose sugar featuring other methods for fluorine introduction have been described. The emphasis of this review is to demonstrate the versatility of synthetic methodology employed in the synthesis of gemcitabine, which is illustrative for general fluorinated carbohydrate synthesis. Some interesting methodology has only been disclosed in the patent literature, of which a selection is covered in this review. In each case only one relevant patent has been cited. While a comprehensive coverage of methods to achieve diastereomer separation falls outside the scope of this review, fair attention is given to the use of particular protecting groups to achieve selective crystallisation, given its relevance in general carbohydrate chemistry. However, the many variations described on the separation of gemcitabine anomers (including precursors and its salts) are not covered in this review.

The original Lilly synthesis is described first. This is then first followed by work towards the synthesis of 2-deoxy-2,2-difluororibose, which is divided in two sections. The first section gives an overview of further optimisations in the fluorinated building block approach, and the second section reviews 2-deoxy-2,2-difluorori- 
<smiles>Nc1ccn(C2OC(CO)C(O)C2(F)F)c(=O)n1</smiles>

gemcitabine

Figure 1. Structure of gemcitabine.

Table 1

Gemcitabine sales figures ${ }^{2}$

\begin{tabular}{llllllll}
\hline & \multicolumn{3}{c}{ Sales (in million \$USD) } & & \multicolumn{2}{c}{ Consumption (in kg) } \\
\cline { 2 - 5 } & \multicolumn{3}{c}{ 12 Months ending } & & & \\
\cline { 2 - 6 } & 2012 & 2011 & Change (\%) & 2012 & 2011 & Change (\%) \\
\hline USA & 103.6 & 405.5 & -74.5 & 1343 & 1433 & -6.3 \\
EU top 5 & 158.8 & 197 & -19.4 & 1621 & 1678 & -3.4 \\
Rest of europe & 43.9 & 60.3 & -27.2 & 643 & 615 & 4.5 \\
Latin america & 2.4 & 2.3 & 4.3 & 12 & 10 & 16.5 \\
Rest of world & 416.4 & 446.5 & -6.7 & 3053 & 2808 & 8.7 \\
Total & 725.2 & $1,111.5$ & -34.8 & 6673 & 6546 & 1.9 \\
\hline
\end{tabular}

bose synthesis starting from carbohydrate/nucleoside precursors. Then, an overview of the methods to introduce the nucleobase is given, again divided in two sections. First, a number of convergent nucleobase syntheses are listed, followed by a linear nucleobase synthesis.

\section{Gemcitabine synthesis}

\subsection{The original synthesis}

The first synthesis of gemcitabine $\mathbf{1}$ was developed in the Lilly research laboratories, and was published by Hertel et al. in 1988 (Scheme 1). ${ }^{6}$

The synthesis starts from enantiopure D-glyceraldehyde $(\boldsymbol{R})-\mathbf{2}$ which can be easily obtained from D-mannitol in 2 steps. ${ }^{7}$ Fluorine introduction was achieved by a building block approach using ethyl bromodifluoroacetate. Reformatsky reaction under standard conditions yielded a 3:1 anti/syn diastereomeric mixture, with the Felkin-Anh product as major diastereomer. Separation of the diastereomers syn- and anti-3 was subsequently carried out by
$\mathrm{HPLC}\left(\mathrm{SiO}_{2}\right)$, which yielded the desired anti-3 in $65 \%$ yield. Subsequent deprotection using Dowex 50 led to concomitant cyclisation to give the $\gamma$-lactone 4 . The remaining free alcohol groups were then protected as TBDMS ethers and subsequent DIBAL-H mediated reduction furnished the key difluororibose intermediate $\mathbf{6}$ in $68 \%$ yield from anti-3.

The fluorination at the ribose 2-position results in a deactivation towards nucleobase introduction, and a better anomeric leaving group was required, such as the corresponding mesylate $\mathbf{7}$, obtained from the lactol as a 1:1 anomeric mixture. Nucleobase introduction was achieved by reaction with silylated cytidine and TMSOTf, which required refluxing in dichloroethane. Subsequent deprotection gave gemcitabine in 50\% yield, but with the undesired $\alpha$-anomer as the major diastereomer (isolated $\alpha / \beta$ ratio $4: 1$ ). The nucleoside anomers were then separated by reverse phase-HPLC, and the identity of the $\beta$-anomer was proven by X-ray crystallography.

Given the strong electron withdrawing effect of the fluorine atoms in the 2-position, an $\mathrm{S}_{\mathrm{N}} 2$ displacement mechanism was expected for the nucleobase introduction. However, given a 1:1 anomeric mixture of mesylate 7 led to a $4: 1 \alpha / \beta$ ratio of nucleosides, the participation of an $S_{N} 1$ pathway cannot be excluded.

\subsection{Synthesis of difluororibose-fluorinated building block approach}

\subsubsection{Reformatsky Reaction}

The Reformatsky reaction starting from ethyl bromodifluoroacetate, introduced by Fried et al., ${ }^{8}$ is a well-established method to introduce a $\mathrm{CF}_{2}$-containing moiety. ${ }^{9}$ Despite the low diastereoselectivity, many groups have used Hertel's Reformatsky procedure for the synthesis of 2-deoxy-2,2-difluororibose. Interestingly, the Reformatsky reaction starting from methyl iododifluoroacetate with D-glyceraldehyde acetonide only gave a 1.8:1 anti/syn ratio (45\% yield). ${ }^{10}$ L-2-Deoxy-2-difluoronucleosides are accessible via the Reformatsky reaction with L-glyceraldehyde acetonide, ${ }^{11}$ which is synthesised from L-gulonolactone in two steps. $^{12}$

An improvement of note was achieved when the zinc was activated with iodine, and the reaction mixture was agitated in an ultrasonic bath under cooling $\left(12 \mathrm{~h}, 10-12^{\circ} \mathrm{C}\right) .{ }^{13}$ An improved yield of $75 \%$ of anti-3 was thus obtained after chromatography (no ratio given at the crude reaction mixture stage).

The L-threose derivative $\mathbf{8}$ has been employed as starting material in the synthesis of gemcitabine and homogemcitabine, also with a Reformatsky reaction as the key step (Scheme 2). ${ }^{14}$ However, no diastereomeric ratio was given. Presumably, the recrystal-
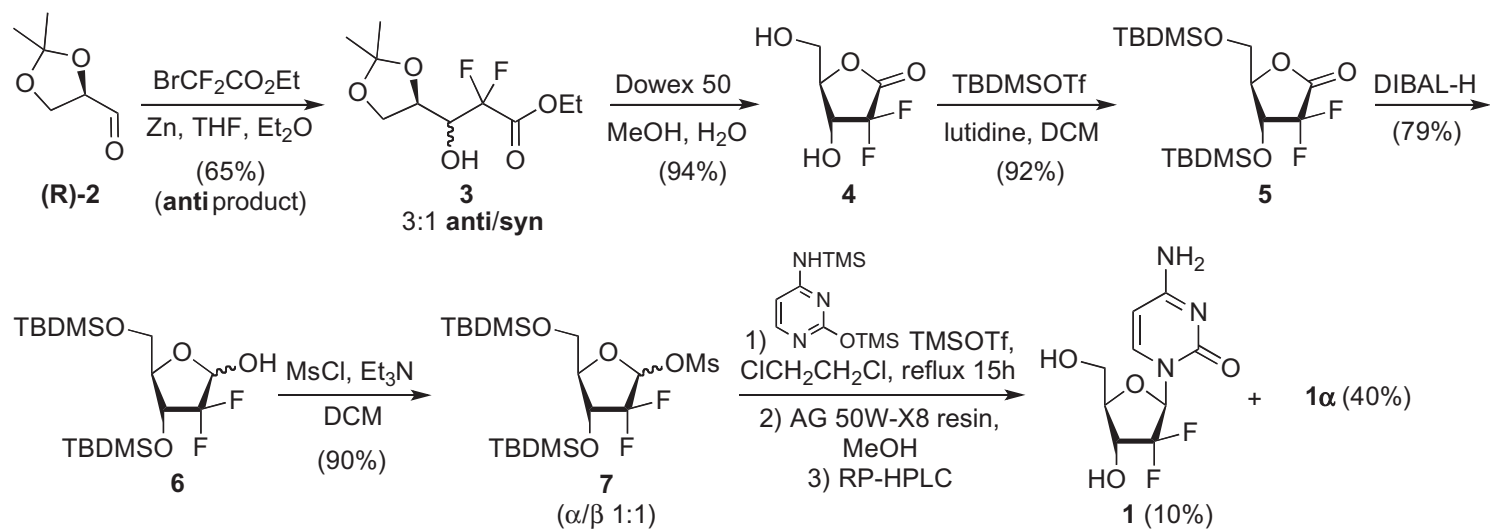


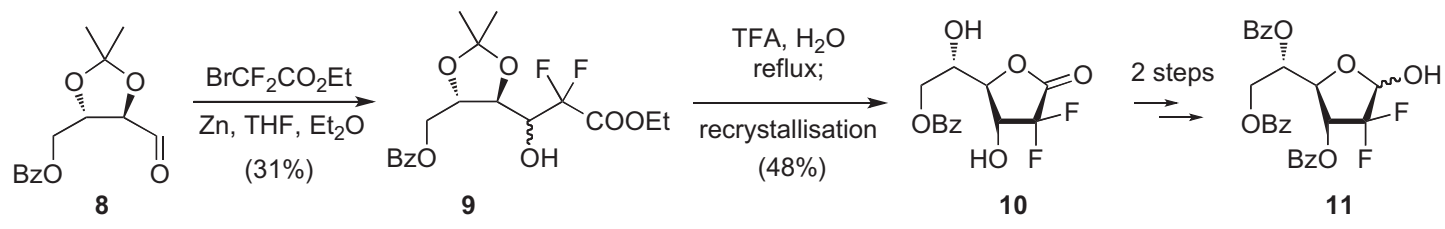

Scheme 2. Reformatsky reaction on L-threose derivative $\mathbf{8}$. ${ }^{14}$

(1)<smiles>CC#CC(F)(F)[C@H](O)[C@H]1COC(C)(C)O1</smiles>

(2) (R)-2

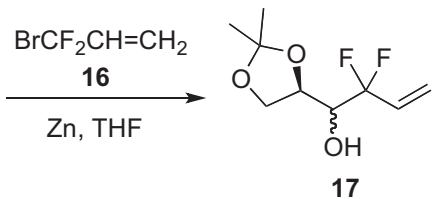

(3.3:1 anti/syn)

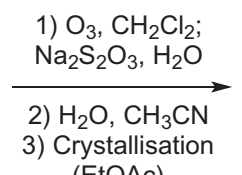

(EtOAc)

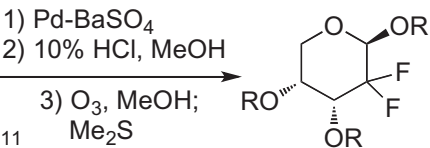

$\mathrm{Ac}_{2} \mathrm{O}, \mathrm{Et}_{3} \mathrm{~N},-14(\mathrm{R}=\mathrm{H})$

DMAP $\longrightarrow 15(R=A c)$

(23\% from anti-13)

Scheme 3. The use of alternative Reformatsky reagents $\mathbf{1 2}$ and $\mathbf{1 6}$ in the synthesis of 2-deoxy-2,2-difluororibose. ${ }^{15,16}$

lisation step after the acetonide hydrolysis/lactonisation sequence allowed separation of the diastereomers, leading to diastereomerically pure 10. Protection and lactone reduction then gave the difluorolactol 11.

In addition to ethyl bromodifluoroacetate as fluorinated building block, 2-deoxy-2,2-difluororibose has also been synthesised using Reformatsky-type reactions with other reagents (Scheme 3). Treatment of bromodifluoroacetylene $\mathbf{1 2}$ with zinc, followed by reaction with D-glyceraldehyde acetonide $(\boldsymbol{R})-\mathbf{2}$, led to the addition product 13 in 50\% yield, as a 3/1 anti:syn ratio (Scheme 3, (1)). ${ }^{15}$ Diastereomeric separation using flash chromatography was possible, and anti-13 was subjected to partial alkyne hydrogenation, ozonolysis/ $\mathrm{Me}_{2} \mathrm{~S}$ treatment, with final protection of the obtained product $\mathbf{1 4}$ as the triacetate $\mathbf{1 5}$.

Alternatively, bromodifluoropropene $\mathbf{1 6}$ has also been used for the synthesis of 2-deoxy-2,2-difluororibose (Scheme 3, (2)). ${ }^{16}$ The addition proceeded in slightly higher diastereoselectivity, and the diastereomers were separated by crystallisation of $\mathbf{1 4}$ by slow evaporation of EtOAc. Both 14 and 15 have been used as intermediates for the synthesis of the lactone $\mathbf{4}$ (see Section 2.3.6).

\subsubsection{Aldol reactions}

A number of reports describe the reaction of glyceraldehyde acetals with difluoroacetate derived enolate species (Scheme 4). The direct formation of lithium enolate $\mathbf{2 3}$ from ethyl difluoroacetate $\mathbf{1 8}$ appears hampered by a dominant Claisen self-condensation side reaction (Eq. 1). ${ }^{17}$ However, starting from $t$-butyl difluorothioacetate 19, Weigel et al. achieved the formation of the less reactive lithium enolate 24 (Eq. 2). ${ }^{17}$ This enolate is formed by adding 19 to a slight excess of LDA at $-78^{\circ} \mathrm{C}$, and is then rapidly ( $2 \mathrm{~min}$ ) reacted with the electrophile. Nevertheless, the Claisen condensation product is still formed in small amounts (10\%), but this could be completely avoided by forming the corresponding ketene silyl O,S-acetal 25 (Eq. 3). Kobayashi et al. achieved the

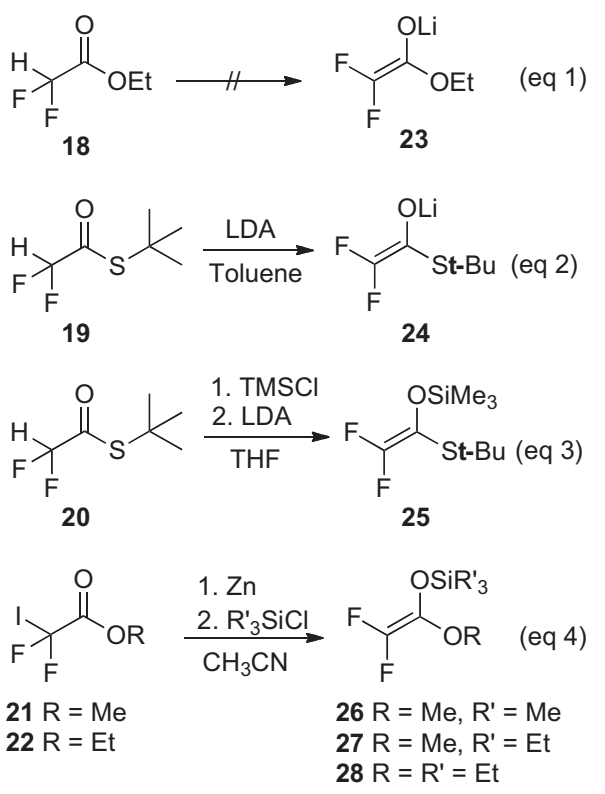

Scheme 4. Synthesis of the difluoroenolate derivatives $\mathbf{2 4 - 2 8} .^{10,17}$

formation of ketene silyl acetals 26-28 via a modification of the Reformatsky conditions, in which a trialkylchlorosilane was included in the reaction mixture before addition of the electrophile (Eq. 4). ${ }^{10}$ The TMS-derivative $\mathbf{2 6}$ proved unstable even at room temperature, but larger silyl groups, such as TES $(\mathbf{2 7}, \mathbf{2 8})$ and TBDMS (not shown) were relatively stable.

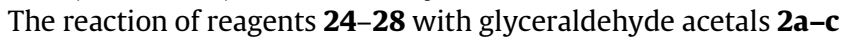
was shown to proceed with high to very high selectivity (Table 2 ). 
Table 2

Aldol-type addition reactions to glyceraldehyde acetals $\mathbf{2}$

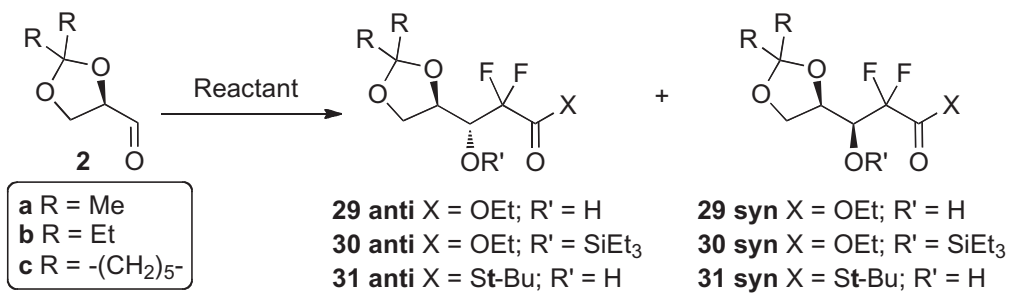

\begin{tabular}{|c|c|c|c|c|c|c|}
\hline Entry & Aldehyde & Reactant & Lewis-acid (equiv) & Product & Yield\% (anti:syn) & Ref. \\
\hline 1 & $2 \mathbf{b}$ & 24 & - & 31b & $64(85: 15)$ & 17 \\
\hline 2 & $2 a$ & 26 & - & $29 \mathbf{a}^{\mathrm{a}}$ & $46(9: 1)$ & 10 \\
\hline 3 & $\mathbf{2 a}$ & 27 & - & $30 \mathbf{a}^{\mathrm{a}}$ & $74(9: 1)$ & 10 \\
\hline 4 & $2 c$ & 28 & - & $30 c$ & $60(85: 15)$ & 18 \\
\hline 5 & $2 c$ & 28 & $\mathrm{BF}_{3} \cdot \mathrm{OEt}_{2}(1)$ & $29 c$ & $47(91: 9)$ & 18 \\
\hline 6 & $2 c$ & 28 & $\mathrm{Me}_{2} \mathrm{AlCl}(1)$ & $29 c$ & $80(78: 22)$ & 18 \\
\hline 7 & $2 c$ & 28 & $\mathrm{TiCl}_{4}(1)$ & $29 c$ & $74(89: 11)$ & 18 \\
\hline 8 & $2 c$ & 28 & $\mathrm{Cp}_{2} \mathrm{TiCl}_{2}(0.1)$ & $30 c$ & $80(90: 10)$ & 18 \\
\hline 9 & $2 c$ & 28 & $\mathrm{Cp}_{2} \mathrm{TiCl}_{2}(1)$ & $30 c$ & $68(>95: 5)$ & 18 \\
\hline 10 & $2 c$ & $28^{\mathrm{b}}$ & $\mathrm{Cp}_{2} \mathrm{TiCl}_{2}(0.1)$ & $30 c$ & $92(91: 9)$ & 18 \\
\hline 11 & $2 c$ & $28^{b}$ & $\mathrm{Cp}_{2} \mathrm{TiCl}_{2}(1)$ & $30 c$ & $84(>95: 5)$ & 18 \\
\hline 12 & $\mathbf{2 b}$ & 25 & $\mathrm{BF}_{3} \cdot \mathrm{OEt}_{2}(2)$ & 31b & $74(95: 5)$ & 17 \\
\hline
\end{tabular}

a $\mathrm{X}=\mathrm{OMe}$.

b Reagent derived from $\mathrm{BrCF}_{2} \mathrm{COOEt}$ (according to Eq. 4).

Unfortunately, the reported selectivities resulting from different methods are not always comparable due to the use of different glyceraldehyde protecting groups, which are known to alter the stereoselectivity of addition reactions to the aldehyde group.

Reaction of $\mathbf{2 b}$ with the lithium enolate $\mathbf{2 4}$ proceeded in $85: 15$ anti:syn ratio in moderate yield (entry 1 ). ${ }^{17}$ Using the ketene trimethyl silyl acetal 26, a low yield of 29a was obtained, if in an enhanced 9:1 ratio (entry 2). ${ }^{10}$ This yield was much improved by using the corresponding 27, which gave 30a in $74 \%$ yield with the same anti:syn ratio (entry 3 ).

The same methodology was applied starting from ethyl iododifluoroacetate $\mathbf{2 2}$ instead of the corresponding methyl ester $\mathbf{2 1}$ to give the silyl ketene acetal $\mathbf{2 8}$. The resulting product $\mathbf{3 0}$ c was obtained in a lower ratio despite a cyclohexylidene acetal protected glyceraldehyde $\mathbf{2 c}$ was used (entry 4). ${ }^{18}$ This lower ratio is difficult to rationalise, as better selectivities are typically obtained with this protecting group compared to the corresponding acetonide $\mathbf{2 a}$.

The reactions involving the ketene silyl acetals 26-28 (entries 2-4) are formally Mukaiyama aldol reactions, and these were achieved without the addition of a Lewis-acid. It was thought that the in situ formed $\mathrm{ZnI}_{2}$ was responsible for activating the aldehyde group.

Matsumura investigated the influence of Lewis acid addition. ${ }^{18}$ In the presence of $\mathrm{BF}_{3} \cdot \mathrm{OEt}_{2}$, a similar anti/syn selectivity was obtained, but in lower yield (entry 5 ). The use of $\mathrm{Me}_{2} \mathrm{AlCl}$ gave an excellent yield, but much reduced selectivity (entry 6), while $\mathrm{TiCl}_{4}$ gave a reasonable yield with restored levels of diastereoselectivity (entry 7). However, much improved selectivities were achieved
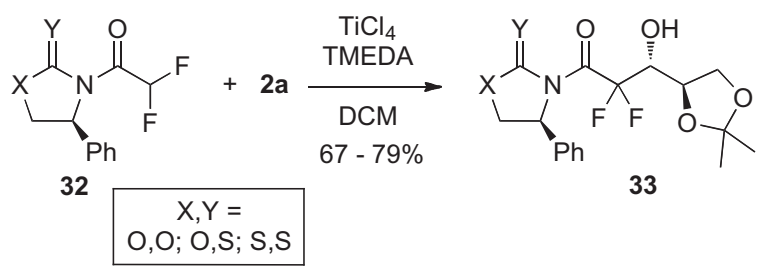

33

Scheme 5. Reaction of $\mathbf{2 a}$ with homochiral enolate derivatives. ${ }^{19}$ when adding a bulky Lewis acid. Hence, with catalytic amounts of $\mathrm{Cp}_{2} \mathrm{TiCl}_{2}$ (entry 8), both yield and diastereoselectivity were enhanced. Interestingly, a stoichiometric amount of the Lewis-acid further improved the diastereoselectivity, but led to a decrease in yield (entry 9). For the same process, but starting from ethyl bromodifluoroacetate instead of ethyl iododifluoroacetate, identical diastereoselectivities but better yields were obtained (entries 10,11). However, a significant drawback of this process for use on large scale is the much higher molecular weight of the Lewis acid compared to the reactants. Finally, Weigel also achieved very high diastereoselectivities when using $\mathrm{BF}_{3} \cdot \mathrm{OEt}_{2}$ in the reaction mediated by the ketene silyl $0, S$-acetal 25 (entry 12). ${ }^{17}$ All syn
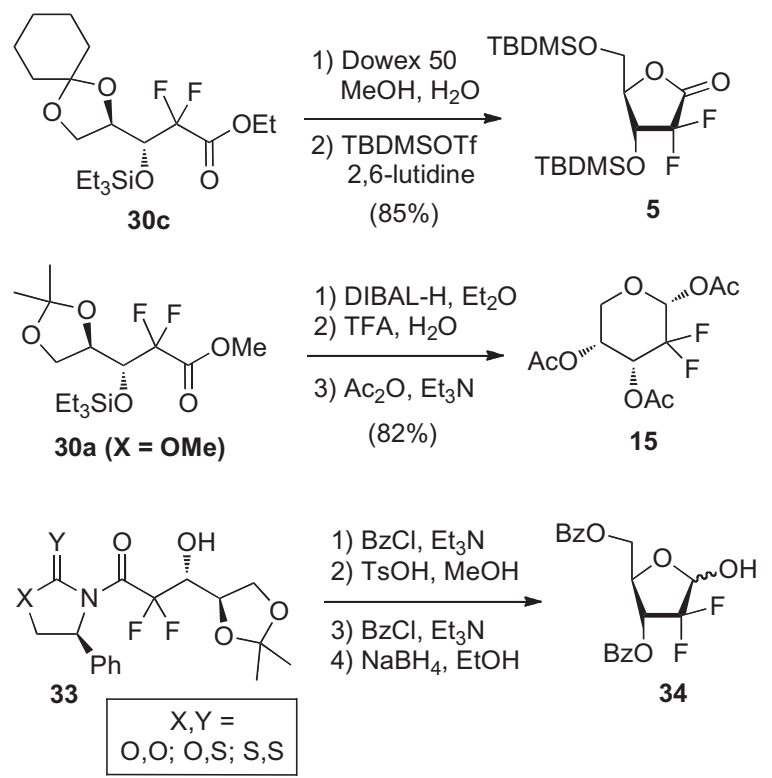

Scheme 6. Further functionalisation of the aldol products to difluororibose derivatives. ${ }^{10,18,19}$ 
and anti diastereomeric mixtures reported above were separable by column chromatography.

All fluorinated reagents described thus far are achiral, with the diastereoselectivities thus originating from the aldehyde chiral centre. An interesting case of a chiral difluoroacetate equivalent 32 was published, using homochiral auxiliaries (Scheme 5). ${ }^{19}$ Unfortunately, no precise diastereoselectivity was described for the formation of $\mathbf{3 3}$, though the obtained product could be used in the next step without purification. Interestingly, reaction of an analogous non-fluorinated acetate-derived homochiral thiazolidinethione reagent with $\mathbf{2 a}$ was reported to give a 13:1 ratio of antiadduct when $\mathrm{PhBCl}_{2}$ /sparteine were used to effect enolisation (not shown). ${ }^{20}$

The products of the various aldol processes were easily converted to difluororibose derivatives (Scheme 6). Silyl-protected product 30c was converted to the lactone $\mathbf{5}$, an intermediate in the Hertel synthesis. ${ }^{18}$ Alternatively, it was shown that ester reduction prior to protection gave the 2-deoxy-2,2-difluororibopyranose triacetate $15 .{ }^{10}$ The conversion of 2-deoxy-2,2-difluororibopyranose derivatives into the required furanose forms is shown in Section 2.3.6. Equally, reduction of the various oxazolidinone, oxazolidine thione and thiazolidine thione auxiliaries with sodium borohydride led to the difluororibose derivative $34 .{ }^{19}$.

\subsubsection{Separation of diastereomers}

The separation of the diastereomers obtained from the Reformatsky reaction by column chromatography or HPLC is impractical on large scale. Several modifications allowing for diastereomeric separation by crystallisation have been developed.

2.2.3.1. Protection as benzoate esters.

In 1992, Chou et al., also from the Lilly research laboratories, reported that protection of the alcohol groups as benzoate esters instead of TBDMS ethers allowed selective crystallisation of the desired 2-deoxy-2,2-difluororibonolactone $\mathbf{3 8}$ on very large scale (Scheme 7 ). ${ }^{21}$

The diastereomeric mixture 3, obtained by a Reformatsky reaction as detailed above, ${ }^{6}$ was benzoylated using $\mathrm{BzCl}$. Despite the deactivation by the adjacent fluorination, a near-quantitative yield was obtained. Hydrolysis of the acetonide gave a mixture of diols 36, which could be cyclised by azeotropic distillation, and the resulting lactone was fully protected using a second benzoylation reaction to give $\mathbf{3 8}$ as a C3-diastereomeric mixture. At this stage, fractional crystallisation from dichloromethane/heptane yielded the diastereomerically pure ribonolactone derivative. This purification was reported to work even on a 2000 gallon scale.

It was mentioned that the lactone group in $\mathbf{3 8}$ was easily solvolysed, requiring great care for the crystallisation. In fact, chromatographic separation was not possible due to silica-gel mediated ring opening.
In the same publication, Chou established lithium t-butoxyaluminium hydride as a superior lactone reducing agent to give the lactol 34. In contrast to reaction with DIBAL-H, no over-reduction with remaining starting material was observed.

2.2.3.2. Substituted benzoate ester protecting groups. Due to the low yield of the recrystallisation of $\mathbf{3 8}$, a number of substituted dibenzoate derivatives were investigated. Cha et al. reported the use of the 3-fluorobenzoyl group as protecting group for lactone 4 to achieve selective crystallisation (Scheme $8(1)$ ). ${ }^{22}$ Conveniently, the crystallisation could be achieved by just adding additional ethyl acetate and hexane, to the ester formation reaction mixture. Hence, starting from a 3:1 diastereomeric mixture of Reformatsky products 3,46\% of lactone $\mathbf{3 9}$ was obtained in a $>98 \%$ purity.

Other substituted benzyl groups, for example $p$-toluoyl, were also shown to be suitable, albeit in a lower overall yield (Scheme 8 $(2)) .^{23}$

Kim et al. developed a separation procedure before cyclisation to the lactone stage (Scheme 9). ${ }^{24}$ The 3:1 diastereomeric mixture of $\mathbf{3}$ was protected as the $p$-phenylbenzoate $\mathbf{4 1}$, before ester hydrolysis to form the potassium salt. Removal of a third of the solvent volume in vacuo resulted in precipitation of the desired anti diastereomer 42 in $70 \%$ yield, contaminated with only $0.1 \%$ of the synbyproduct. Cyclisation and 5-O-benzoylation gave, after another recrystallisation from ether/hexane, the lactone $\mathbf{4 3}$ free from syndiastereomer, in $\mathbf{7 2} \%$ yield. Reduction of $\mathbf{4 3}$ using Chou's procedure then gave 44.

A variation on this purification was published by $\mathrm{Xu}$ et al., who noted the apparent instability of the potassium salt $\mathbf{4 2} .{ }^{25}$ Instead, TFA-mediated acetonide hydrolysis of $\mathbf{4 1}$, followed by lactone formation through azeotropic distillation from toluene gave $\mathbf{4 3}$ as a 3:1 diastereomeric mixture, and purification was achieved by recrystallisation from toluene/hexane, leading to a 50:1 mixture of C3-diastereomers $\mathbf{4 3}$ in 52\% overall yield from $\mathbf{4 1}$ (not shown).

2.2.3.3. Protection as cinnamoyl ester. Jiang et al. ${ }^{26}$ introduced the cinnamoyl protecting group for the lactone $\mathbf{4}$ to obtain the crystalline lactone $\mathbf{4 5}$ (Scheme 10). Selective crystallisation from toluene allowed isolation of $\mathbf{4 5}$ in both high purity (97.1\%) and ee $(99.3 \%)$, in $43 \%$ yield. The lactone was then reduced and used directly in the nucleoside introduction protocol (see below).

2.2.3.4. Protection as phenyl carbamoyl. The use of a carbamoyl group at the 3-position was shown to increase the anomeric ratio in the nucleobase introduction step (see below). While in this case the required substrate was prepared from the corresponding dibenzoate $\mathbf{4 6}$, Naddaka et al. showed that the same protecting group could be used to achieve separation of the

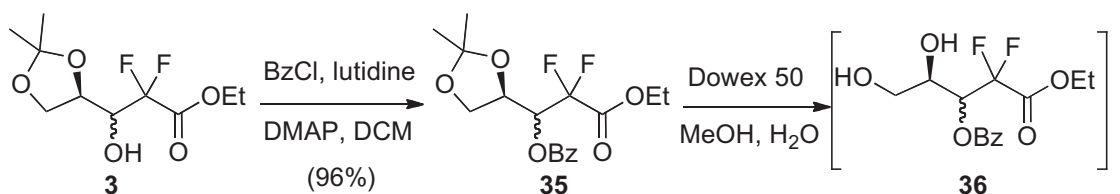

(ratio not specified)

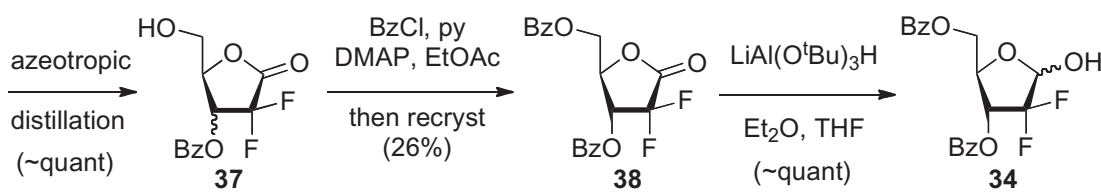


(1)<smiles>CCOC(=O)C(F)(F)[C@H](O)C1COC(C)(C)O1</smiles>

(2)<smiles>CCOC(=O)C(F)(F)[C@@H](O)C1COC(C)(C)O1</smiles>

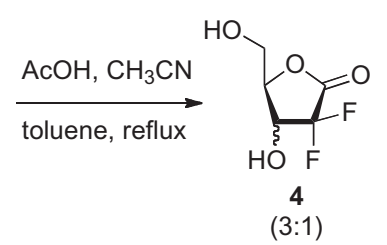

$(3: 1)$

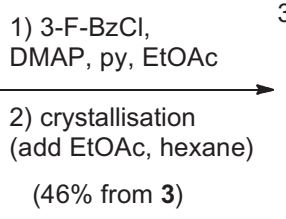

$(46 \%$ from 3$)$

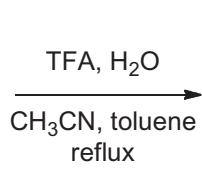

$\sum_{4}^{\mathrm{O} O}=\mathrm{F}$

$(3: 1)$

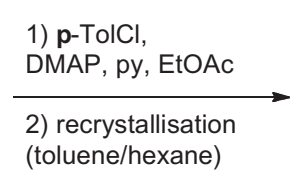

(25\% from 3$)$
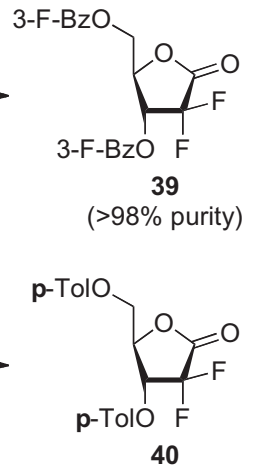

(>99\% purity)

Scheme 8. Diastereomeric resolution using substituted benzoate ester protection. ${ }^{22}$

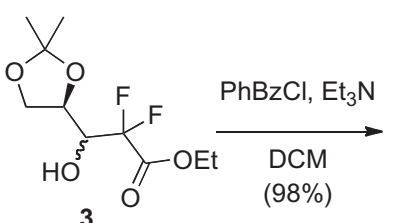

(3:1 anti/syn)

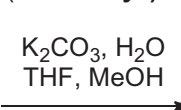

then precipitate $(70 \%)$

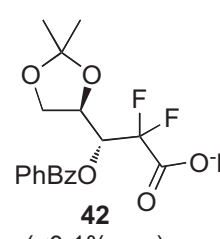

$(<0.1 \%$ syn $)$<smiles>O=C(OCc1ccccc1)OCC1OC(=O)C(F)(F)C1OP</smiles>

43

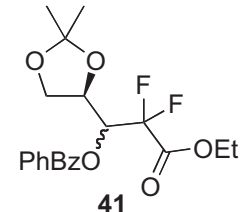

(3:1 anti/syn)
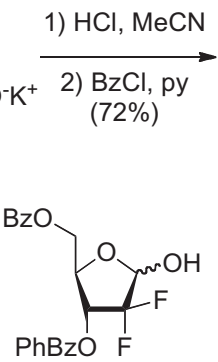

44
Scheme 9. Diastereomeric resolution using $p$-phenylbenzoate protection. ${ }^{24}$<smiles>O=C(Cl)/C=C/[PH](=O)[O-]</smiles>

Scheme 10. Cinnamoyl mediated diastereomeric resolution. ${ }^{26}$

Reformatsky diastereomers by selective crystallisation (Scheme 11). ${ }^{27}$ The thus obtained erythro diastereomer 47 could then be cyclised after treatment with acid, and removal of water by azeotropic distillation.

2.2.3.5. Derivatisation with $\alpha$-methyl benzylamine. Park et al. ${ }^{26}$ devised an alternative separation method based upon selective recrystallisation of an amide derivative. The derivatisation of the ester 3 (Scheme 12) with an optically pure amine such as $(S)$ 1-phenyl ethanamine $\mathbf{4 8}$ produces a mixture of amides from which the desired anti diastereomer $\mathbf{4 9}$ can be recrystallised from hexane or hexane/EtOAc in 54\% yield. Protection of the $3-\mathrm{OH}$ as benzoate ester $\mathbf{5 0}$ (or 2-naphthoyl ester) is then followed by lactonisation
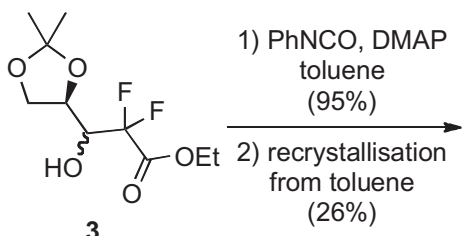

2.6:1 anti/syn $(26 \%)$
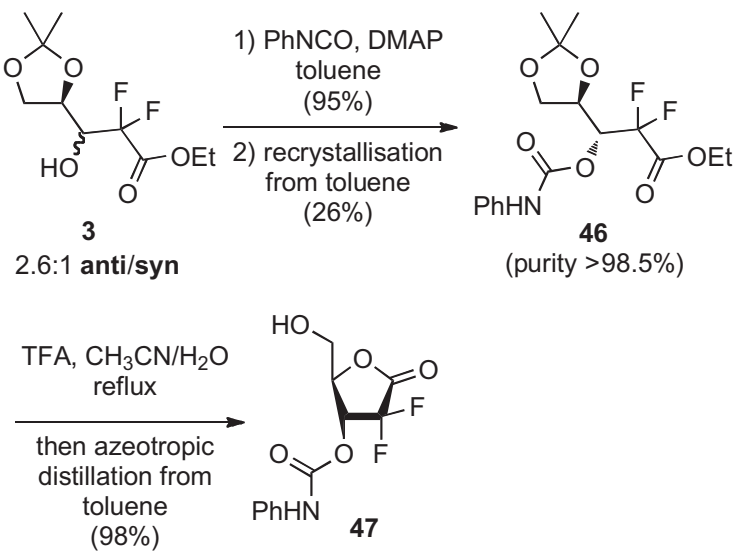

(purity $>98.5 \%$ )

Scheme 11. Phenyl carbamoyl mediated diastereomeric resolution. ${ }^{27}$
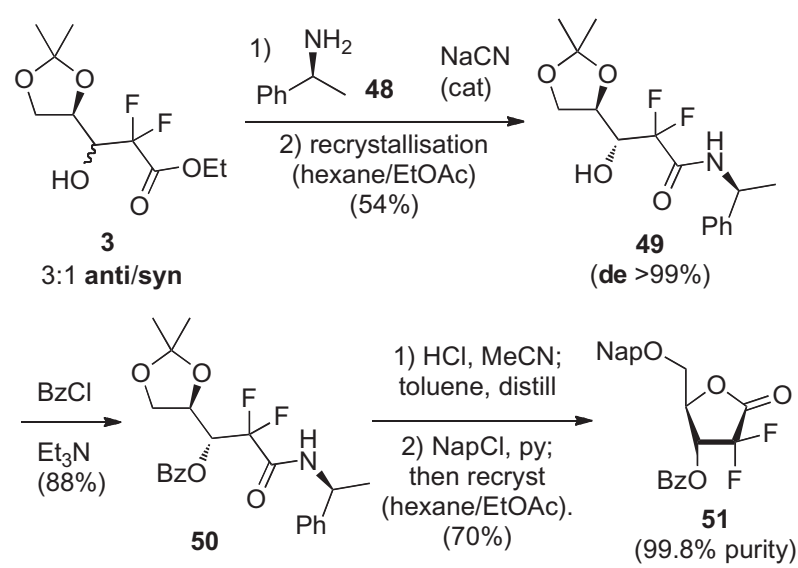

Scheme 12. Resolution with (S)-phenyl ethanamine $48 .^{28}$

and further protection of the 5-OH as 2-naphthoyl (or benzoyl) ester 51. The lactone is obtained in $99.8 \%$ purity (less than $0.2 \%$ of other isomers). Reduction to the lactol is then effected with lithium tri-t-butoxyaluminium hydride (not shown).

\subsection{Synthesis of difluororibose-fluorination of carbohydrate derivatives}

There are a number of reports describing the synthesis of 2deoxy-2,2-difluororibose where fluorine introduction is achieved 

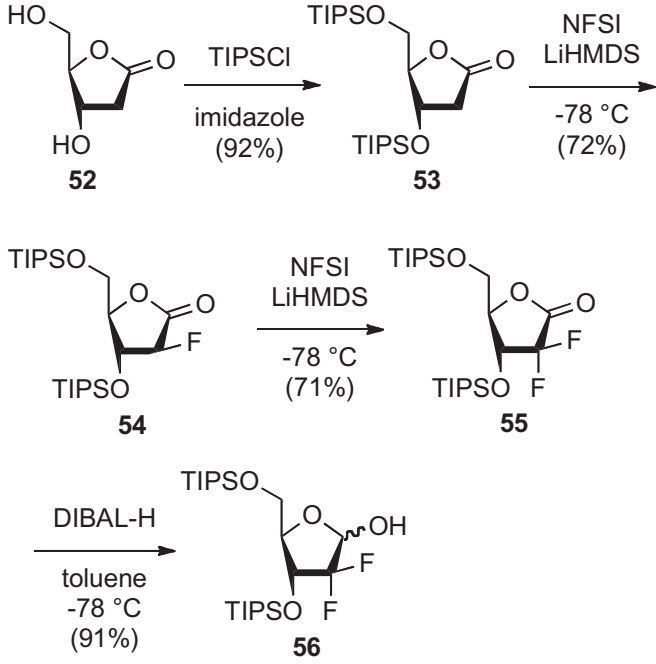

Scheme 13. Synthesis of difluororibose by direct fluorination of 2-deoxy-D -ribonolactone $\mathbf{5 2 .} .^{29}$
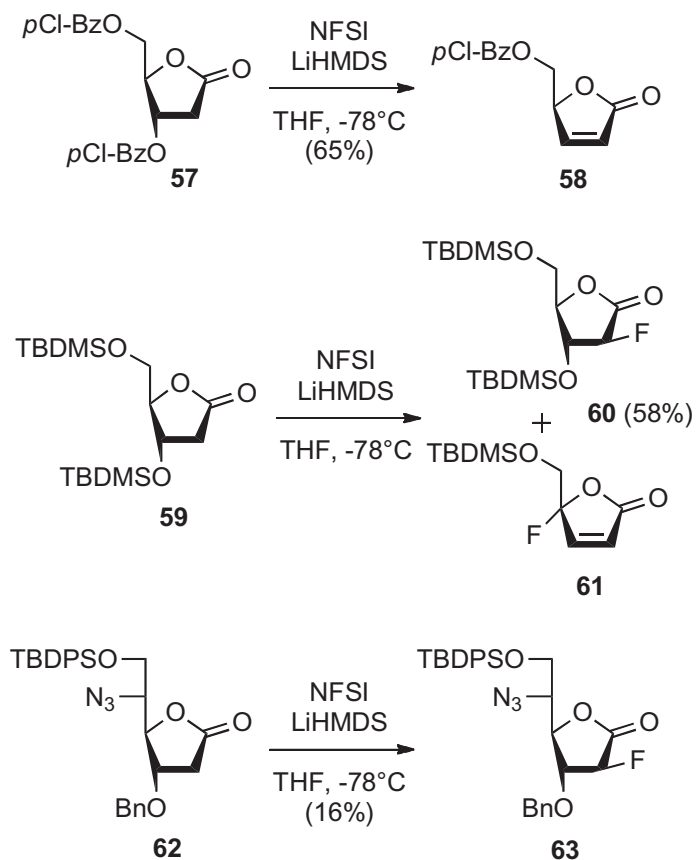

Scheme 14. Electrophilic fluorinations on differently protected 2-deoxylactones. ${ }^{29}$

via fluorination of carbohydrate or nucleoside precursors. Starting from carbohydrate precursors, the synthesis is typically aimed at the difluororibofuranose isomer, ready for nucleobase introduction. However, some reports describe the conversion of the 2,2difluororibopyranose form, which is the more stable isomer, to the required furanose form.

\subsubsection{Direct fluorination of 2-deoxyribonolactone}

Sauve and Cen developed a method to obtain the protected difluororibose $\mathbf{5 6}$ from the readily available 2-deoxy-D-ribonolactone 52 (Scheme 13). ${ }^{29}$ This method does therefore not require stereoselective transformations or separation of diastereomers to obtain the desired stereochemistry at $\mathrm{C} 3$ and $\mathrm{C} 4$, which is an advantage over many of the methods described above for the synthesis of gemcitabine. ${ }^{30}$

Protection of the alcohol groups in 2-deoxy-D-ribonolactone 52 is followed by a diastereoselective electrophilic fluorination of the resulting lactone $\mathbf{5 3}$ with NFSI, to give the monofluoroarabinolactone 54 in $72 \%$ yield. The diastereoselectivity of this reaction was attributed to the steric bulk of the $\mathrm{O} 3$ silyl protection preventing a syn attack by the fluorinating agent. In contrast, the same fluorination reaction on a corresponding 2,3-dideoxylactone proceeds with the opposite diastereoselection, leading to the $\alpha$-fluorolactone (not shown). ${ }^{31}$ From 54, second electrophilic fluorination, again using NFSI, furnished the difluorinated lactone $\mathbf{5 5}$ in 71\% yield. Lactol $\mathbf{5 6}$ is then obtained via DIBAL reduction in excellent yield.

The success of the fluorination reaction depends on the suppression of a competing elimination side reaction of the intermediate lactone lithium intermediate. As could be expected, a 3-O-ester protecting group only gave the elimination product $\mathbf{5 8}$ (Scheme 14), but changing to a TBDMS ether gave $58 \%$ of the desired 2-deoxy-2-fluoroarabinolactone $\mathbf{6 0}$. However, the formation of $\mathbf{6 1}$ in that reaction showed that competitive elimination is still occurring. It is worth noting that a 3-O-benzyl ether, as in $\mathbf{6 2}$, was not superior as a protecting group, which based on $\mathrm{p} K_{\mathrm{a}}$ considerations (alcohol $\mathrm{p} K_{\mathrm{a}}=16$ versus silanol $\mathrm{p} K_{\mathrm{a}}=11$ ) is difficult to understand. However, a bulky 3-O-TIPS ether as in 53 (see Scheme 13) proved to be a very successful substituent to achieve successful fluorination.

Instead, Sauve and Cen postulated that increasing the steric bulk of the protecting group at 3-OH would force the ring conformation such that $\mathrm{O} 3$ is in a pseudoequatorial position, lowering its propensity for elimination. An MM2 minimisation of the lithium enolate of $\mathbf{5 3}$ confirmed this.

\subsubsection{Synthesis from D-ribose}

A short synthesis of a 2-deoxy-2,2-difluororibose precursor was reported by Gong (Scheme 15). ${ }^{32}$ The commercially available 1-O-acetyl-2,3,5-tri-O-benzoyl- $\beta$-d-ribofuranose 64 was subjected to an anomeric bromination/ester migration sequence to give the $1,3,5$-tri-O-benzoyl- $\alpha$-D-ribofuranose $\mathbf{6 5}$. ${ }^{33}$ Oxidation of the free 2-OH group to the ketone then allowed for fluorination with a DAST-HF.Et ${ }_{3} \mathrm{~N}$ mixture to give 67 , which was then used directly for the nucleobase introduction. The use of DAST alone in the fluorination step was reported to give low yields.

\subsubsection{Synthesis from D-mannose}

The group of Castillon has described two approaches for the synthesis of 2-deoxy-2,2-difluororibose, both relying on the direct fluorination of a 3-ulose intermediate. ${ }^{34}$ The first approach started from methyl- $\alpha$-D-mannopyranoside $\mathbf{6 8}$, which was converted to the required ulose $\mathbf{7 0}$ using a known procedure (Scheme 16). ${ }^{35}$

Reaction of $\mathbf{7 0}$ with DAST gave the 2,2-difluorinated $\mathbf{7 1}$ in 70\% yield. The 4,6-di-O-benzylidene acetal was then hydrolysed to allow the desired benzoate protection. Conversion of the anomeric methyl acetal to the corresponding selenide, followed by oxidative elimination gave the glycal 73. Reductive ozonolysis and subsequent hydrolysis then furnished the desired protected difluororibose 34

\subsubsection{Synthesis from $D$-glucose}

A second approach described by Castillón relied on fluorination of ulose 74, still oxygenated in the 2-position (Scheme 17). ${ }^{34}$

The ulose $\mathbf{7 4}$ was synthesised from D-glucose in 4 steps, involving sequential protections of the anomeric position, the 4- and $6-\mathrm{OH}$ positions, and finally the $2-\mathrm{OH}$ position (not shown). Oxidation of the remaining $3-\mathrm{OH}$ then gave $\mathbf{7 4}$, followed by DAST-mediated fluorination to give $\mathbf{7 5}$. The benzylidene acetal 


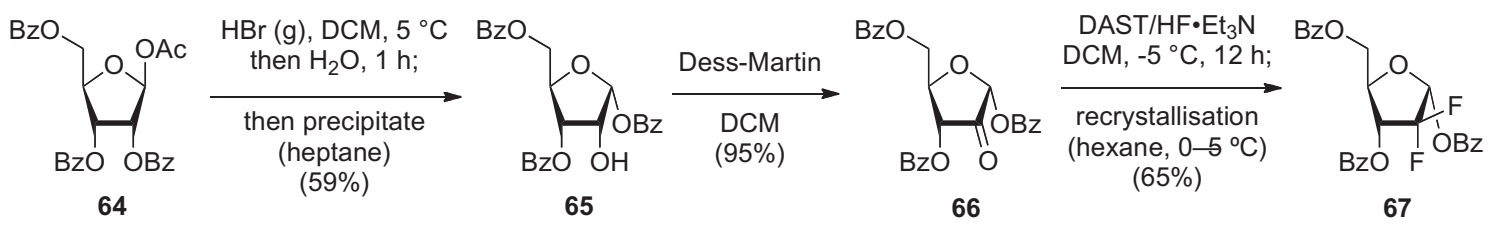

Scheme 15. Direct fluorination of a ribose derivative to give a gemcitabine precursor. ${ }^{32}$
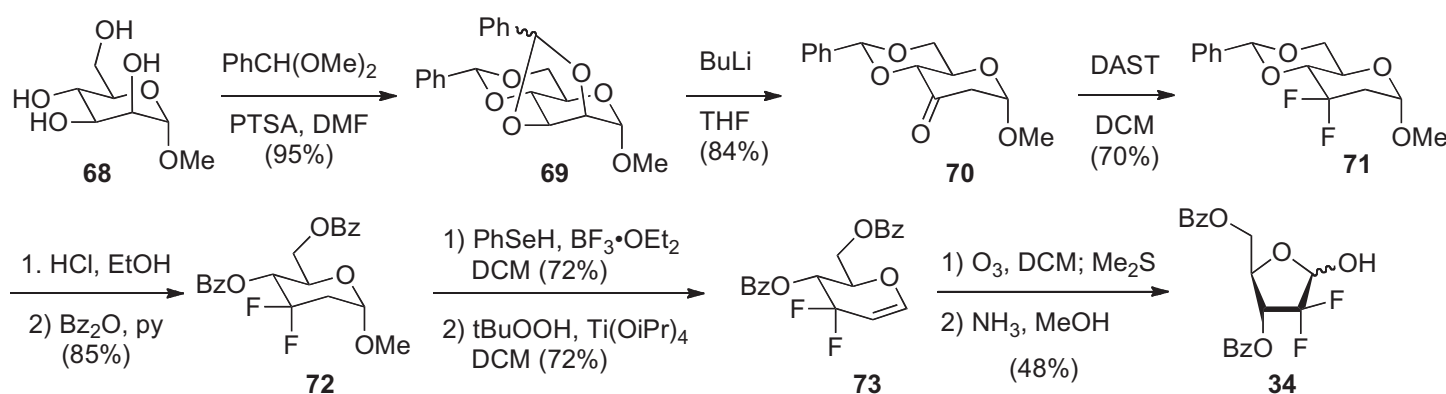

Scheme 16. Synthesis of protected 2,2-difluororibose 34 from D-mannose. ${ }^{34}$
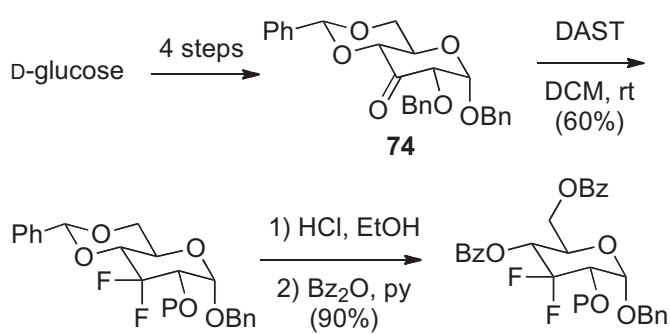

$75(\mathrm{P}=\mathrm{Bn})$
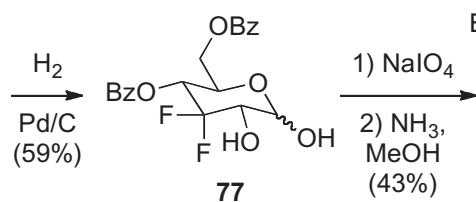

$$
76(P=B n)
$$

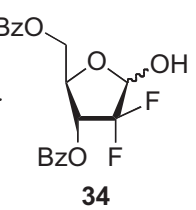

Scheme 17. Synthesis of protected 2,2-difluororibose $\mathbf{3 4}$ from D-glucose. ${ }^{34}$

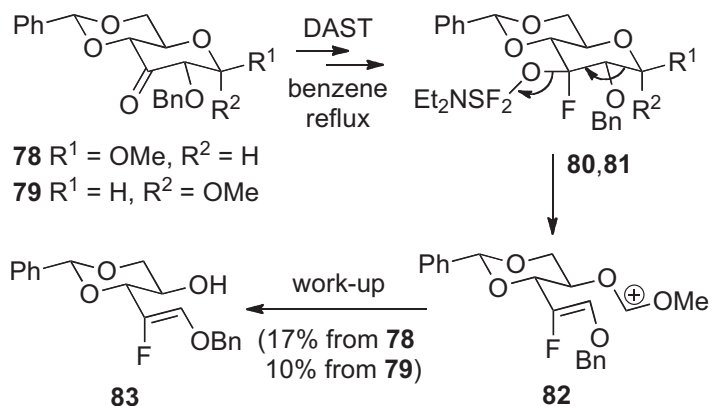

Scheme 18. Fragmentation side reaction in the fluorination of 3-uloses. ${ }^{36}$

was then hydrolysed to allow for the installation of the desired benzoates, to give the difluorosugar $\mathbf{7 6}$ in 90\% yield. Hydrogenation removed the benzyl protecting groups in 59\% yield to give $\mathbf{7 7}$, enabling sodium periodate mediated cleavage of the diol. Subsequent hydrolysis of residual 4-O-formate ester by methanolic $\mathrm{NH}_{3}$ furnished the desired protected difluororibose $\mathbf{3 4}$ in $43 \%$ yield. It was reported that when the methyl glycoside derivative was used, anomeric deprotection was low-yielding.

Interestingly, the DAST-mediated fluorination of 3-uloses was first studied in benzene, which gave lower yields of the corresponding difluorosugars (40-48\%, not shown). This was found to be due in part to the formation of the Grob-fragmentation product $\mathbf{8 3}$, the proposed mechanism of which is shown in Scheme $18 .^{36}$

\subsubsection{Synthesis from 1,6 -anhydro- $\beta$-D-glucose}

Gong also reported the synthesis of 2-deoxy-2,2-difluororibose from 1,6-anhydro- $\beta$-D-glucose 84 (Scheme 19). ${ }^{37}$ Selective protection as the 2,4-di-O-TMS ether gave a near quantitative yield of 85, which was oxidised with Dess-Martin periodinane. The silyl ethers were subsequently removed to give ulose $\mathbf{8 6}$. Reprotection of $\mathrm{O} 2$ and $\mathrm{O} 4$ as methyl ethers allowed for the fluorination of ulose 87 with DAST in the presence of DMPU-HF. The use of both DAST and DMPU-HF not only gave an increased yield of the difluorosugar $\mathbf{8 8}$, but also allowed the reaction time to be significantly shortened. Hydrolysis in strong acidic medium gave 3-deoxy-3,3-difluoroglucose 89. Periodate oxidation was reported to selectively cleave the $\mathrm{C} 1-\mathrm{C} 2$ bond to give 2-deoxy-2,2-difluororibose 14 (shown here in the pyranose form).

\subsubsection{Conversion of 2-deoxy-2,2-difluororibopyranose to the furanose isomer}

Given the higher stability of pyranoses compared to the corresponding furanoses, pentose derivatives with unprotected 4- and $5-\mathrm{OH}$ groups typically adopt the pyranose form. While unprotected 2-deoxy-2,2-difluororibose can be found depicted in the literature both as the pyranose and the furanose forms, it is generally accepted that also in this case, the pyranose form is the most stable tautomer. While the structure of 2-deoxy-2,2-difluororibopyranose was reported to be confirmed by X-ray crystallography, ${ }^{38}$ the structure has not been deposited to the Cambridge Crystallographic database.

The isomerisation of the pyranose to the corresponding furanose form can be achieved, generally, by certain alcohol protection protocols, and this has been demonstrated in the context of gemcitabine synthesis. 

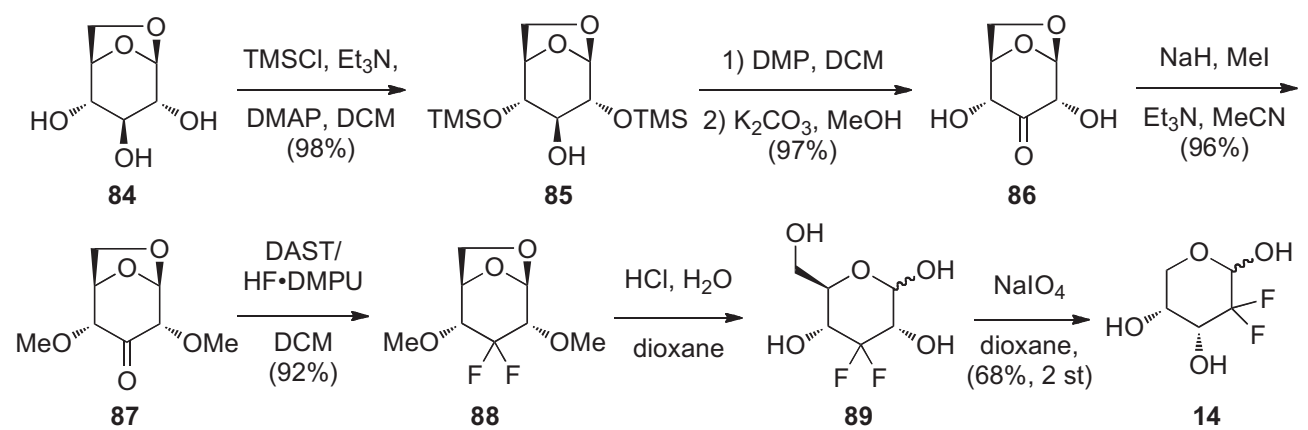

Scheme 19. Synthesis from 1,6-anhydro- $\beta$-D-glucose $\mathbf{8 4} .^{37}$

(1)<smiles>O[C@H]1CO[C@H](O)C(F)(F)[C@@H]1O</smiles>

(2)

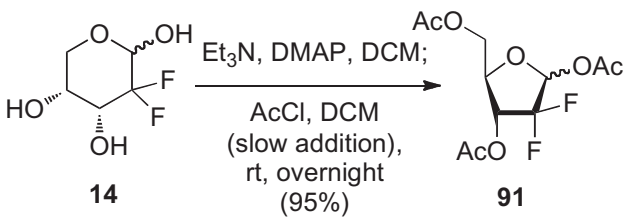

Scheme 20. Pyranose to furanose isomerisation of difluororibose $\mathbf{1 4}$ by 5-OH tritylation ${ }^{39}$ or acetylation. ${ }^{37}$

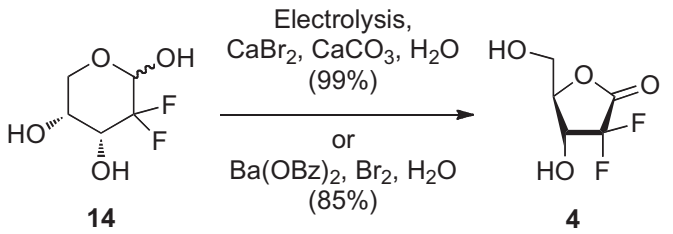

Scheme 21. Ring isomerisation by $\mathrm{C} 1$ oxidation. ${ }^{38}$

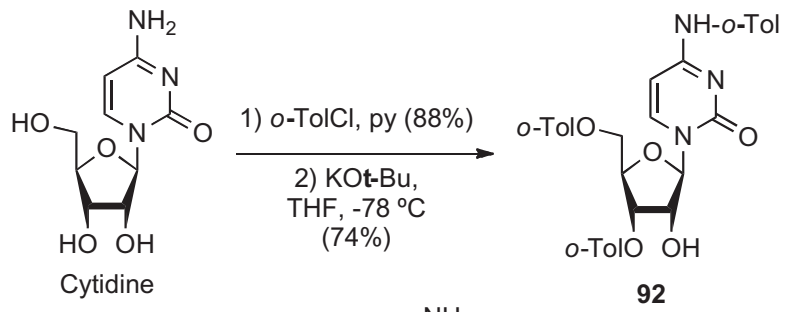

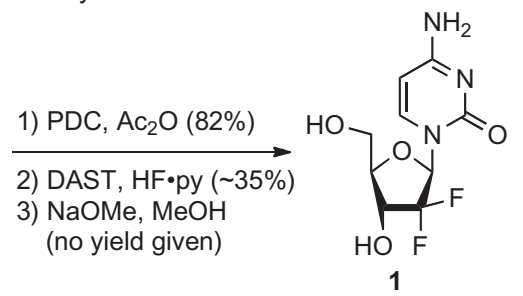

Scheme 22. Synthesis of gemcitabine via fluorination of cytidine. ${ }^{40}$
Wirth used trityl protection to obtain the difluororibofuranose isomer 90 (Scheme 20, (1)). ${ }^{39}$ The isomerisation is possible due to the presence of a pyranose-furanose equilibrium in solution phase, and the much faster tritylation of primary alcohols compared to secondary ones. A crude yield of $47 \%$ was reported, which, after purification by flash chromatography dropped to $19 \%$.

A similar isomerisation was achieved by an acetylation reaction (Scheme 20, (2)). Though there are many reports describing the acetylation of 2-deoxy-2,2-difluororibopyranose 14 to give the corresponding triacetate in the pyranose form, ${ }^{10,15,38}$ the outcome reported here, with slow addition of $\mathrm{AcCl}$, can be understood by initial acetylation of the primary $5-\mathrm{OH}$ group before reaction of the secondary $\mathrm{OH}$ groups. Unfortunately no spectral details of $\mathbf{9 1}$ were provided, though subsequent conversion to gemcitabine is clearly proof of structure.

As mentioned before, a $\gamma$-lactone is more stable than a $\delta$-lactone, which has already been exploited in the first Hertel synthesis of gemcitabine. In a patent describing a difluororibose recycling method starting from the undesired gemcitabine $\alpha$-anomer, Nagarajan published two oxidation protocols in which the difluoropyranose is converted to the corresponding $\gamma$-lactone $\mathbf{4}$ (Scheme 21) in excellent yield. ${ }^{38}$ The lactone is water-soluble, and was obtained in pure form after several lyophilisation cycles.

\subsubsection{Fluorination of nucleoside derivatives}

Gemcitabine has also been obtained via fluorination of 2-keto nucleoside derivatives. Kjell reported a synthesis from cytidine (Scheme 22). ${ }^{40}$ Full protection of all alcohol groups as well as the cytosine amino group, followed by regioselective deprotection at the 2'-position gave 92, which could then be oxidised and fluorinated with DAST and HF-pyridine. As in the Chen synthesis, ${ }^{37}$ DAST alone does not effect the fluorination, in this instance the reaction does not proceed in the absence of HF-pyridine. Finally NaOMe mediated deprotection furnishes gemcitabine.

An interesting synthesis via fluorination of a nucleoside derivative was reported by Noe et al. (Scheme 23). ${ }^{14}$ Starting from 1,2isopropylidene allofuranose $\mathbf{9 3}$, protective group manipulations led to 94, which acted as substrate for the nucleobase introduction. No anomeric ratio was given, but presumably chromatography after subsequent selective removal of the phenoxyacetate group led to anomerically pure product, which after oxidation gave $\mathbf{9 5}$. Fluorination, again with a DAST-HF.py mixture was then followed by full deprotection to give homogemcitabine. Gemcitabine was then obtained by periodate cleavage of the side-chain diol, followed by reduction.

\subsection{Nucleobase introduction-direct coupling}

Nucleobase addition methods, such as the Hilbert-Johnson and Vorbruggen protocols, are disfavoured in the synthesis of gemcitabine due to the highly electron withdrawing nature of the difluoro 

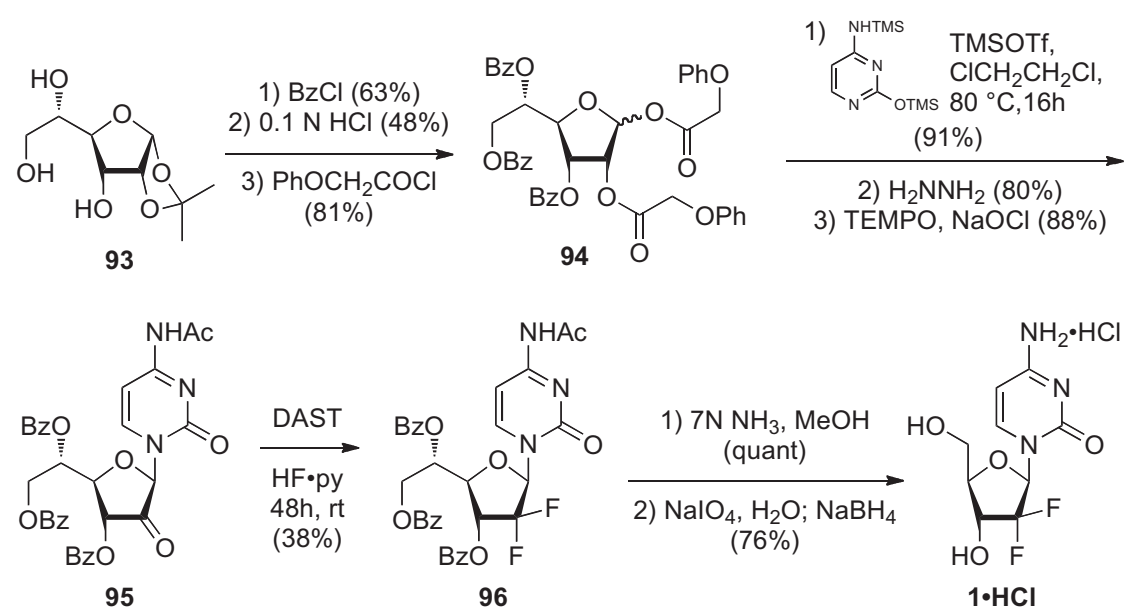

Scheme 23. Synthesis of gemcitabine (and homogemcitabine) via fluorination of a homocytidine derivative. ${ }^{14}$

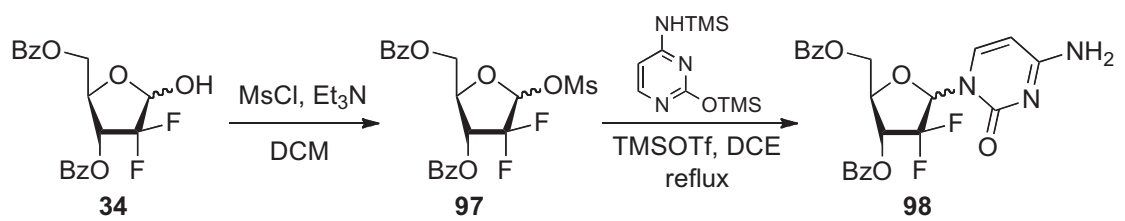

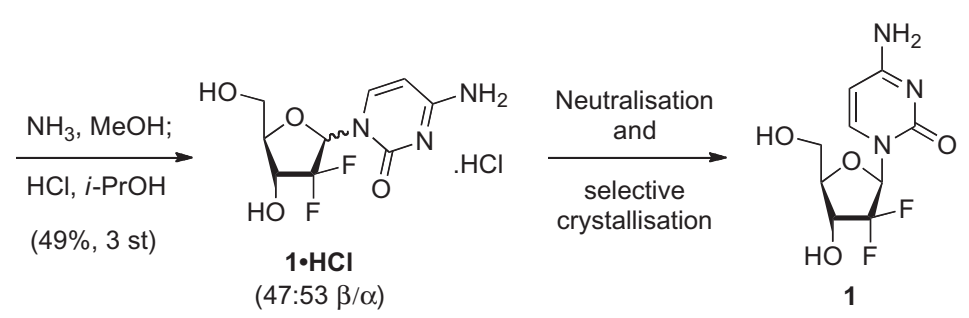

Scheme 24. Completion of Chou's gemcitabine synthesis. ${ }^{21}$

moiety next to the anomeric centre. Hence this reaction has been subject to extensive optimisation, not only with regard to yield/ conversion, but also to anomeric selectivity. The selected coverage in this review is focused on the various donor systems that have been used to achieve nucleobase introduction. Many crystallisation protocols have been described in order to obtain gemcitabine, or its hydrochloride salt, in high anomeric purity. However, though some examples of crystallisation protocols are given, comprehensive coverage of the anomeric purification protocols falls outside the scope of this review.

\subsubsection{Mesylate leaving group}

In the original Hertel synthesis (Scheme 1 ), ${ }^{6}$ nucleobase introduction was achieved by displacement of a mesylate leaving group by a disilylated cytosine nucleophile, on a 3,5-disilylated (TBDMS) difluororibose sugar. Clearly the obtained anomeric selectivity (favouring the undesired $\alpha$-anomer in a $4: 1$ ratio), was unsatisfactory. Interestingly, the anomeric selectivity was improved (to $1: 1$ ) when the mesylate of the corresponding 3,5-di-O-triisopropylsilyl (TIPS) protected difluororobose sugar was used. ${ }^{30}$ This was also the ratio obtained by Chou et al., starting from dibenzoylated difluororibose (Scheme 24), after deprotection. ${ }^{21}$ Pure gemcitabine was then obtained by selective crystallisation.

The optimisation of the anomeric selectivity has been thoroughly investigated. ${ }^{41}$ It was found that lowering the temperature in the mesylation reaction favoured formation of the $\alpha$-mesylate. At $19{ }^{\circ} \mathrm{C}$ a $2: 1 \alpha / \beta$ ratio is obtained while a $4.4: 1 \alpha / \beta$ ratio was obtained when the reaction is carried out at $-83^{\circ} \mathrm{C}$. No mention is made of the effect the lowered temperature has upon the yield of the reaction. Alternatively, $\beta$-mesylate could be equilibrated by reaction with $\mathrm{N}, \mathrm{N}$-dimethylbenzylammonium methanesulfonate at reflux temperature, to obtain a 2.3:1 $\alpha / \beta$ mixture of mesylates. ${ }^{41}$

While initial investigations into the mesylate displacement gave a 1:1 mixture of protected nucleoside $\mathbf{9 8}$ regardless of the anomeric ratio of mesylate $\mathbf{9 7},{ }^{21}$ further investigation did give anomerically enriched $\beta$-nucleoside 98 starting from $\alpha$-enriched mesylate 97. ${ }^{41}$ Starting from the $\alpha$-anomer, the best reported method was the use of bis-silylated cytosine in anisole at $115^{\circ} \mathrm{C}$, yielding $79.5 \%$ of the protected nucleoside $\mathbf{9 8}$ as a $7.3: 1 \beta / \alpha$ mix-<smiles>COC(=O)OCC1OC(OC(=O)OCc2ccccc2)C(F)(F)C1OC(N)=O</smiles>

Figure 2. 3-O-Carbamoyl derived substrate. ${ }^{44,13}$ 


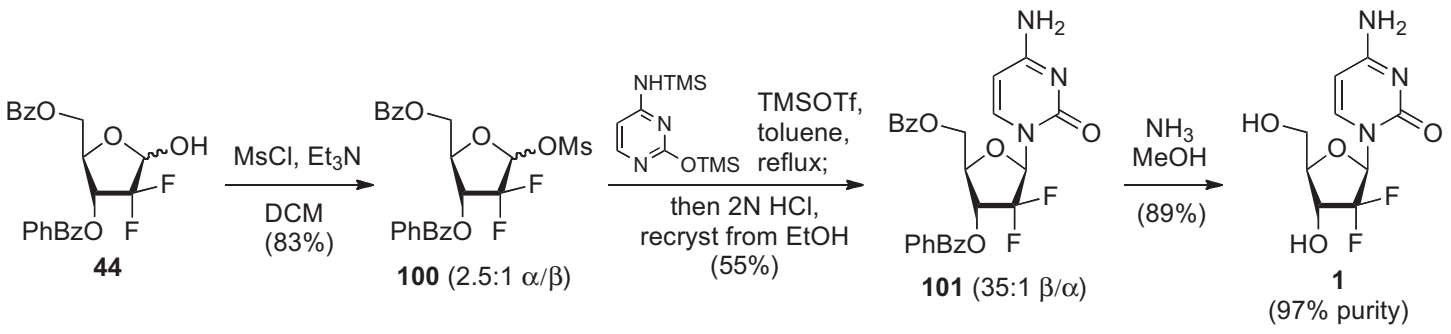

Scheme 25. The use of the 4-phenylbenzoate protecting group to achieve selective crystallisation of the $\beta$-anomer. ${ }^{25}$

ture. Alternatively nucleoside $\mathbf{9 8}$ could be synthesised with very high $(>14: 1 \beta / \alpha)$ anomeric selectivity when treated with bis-silylcytosine in $\mathrm{MeCN}$ at $75{ }^{\circ} \mathrm{C}$ in the presence of caesium sulfate or barium triflate, however with a significant reduction in yield ( 25\%).

A later patent from $\mathrm{Chou}^{42}$ describes the development of a solventless protocol for nucleobase addition. Interestingly, while starting from the $\beta$-mesylate, displacement with silylated nucleobase gave predominantly the $\alpha$-nucleoside (1:6-7 $\beta / \alpha$ ratio); from the $\alpha$-mesylate, the $\beta$-nucleoside was the major anomer, but in lower ratios (up to $4: 1 \beta / \alpha$ ). Kjell $^{43}$ described that addition of certain salts in the glycosylation reaction increased the anomeric selectivity. The best selectivity $(14.9: 1 \beta / \alpha)$ was obtained with the use of $\mathrm{Cs}_{2} \mathrm{SO}_{4}$, but with a poor yield of $24 \%$. Use of the caesium salt of triflic acid however, furnished a $6.7: 1 \beta / \alpha$ mixture of the protected nucleoside $\mathbf{9 8}$ in $70 \%$ yield. It should be noted that in these cases pure $\alpha$-mesylate $\mathbf{9 7}$ was employed.

The anomeric ratio could also be improved by using a carbamoyl protecting group at the 3-position. For example, nucleobase introduction with 99 (Fig. 2) led to a $\beta / \alpha$ ratio of around 1.5:1, compared to a $1: 1.5$ ratio when the corresponding dibenzoate 97 is used under the same conditions. ${ }^{44}$

Some of the protecting groups that were introduced to separate the diastereomers arising from the Reformatsky reaction proved also useful to separate the nucleoside anomers by crystallisation. ${ }^{25}$ For example, the 4-phenylbenzoate protected difluororibose derivative 44 (Scheme 25). Mesylation led to a 1:2.5 $\beta / \alpha$ ratio of anomers. Nucleobase introduction yielded a $1.8: 1 \quad \beta / \alpha$ ratio of anomers which upon nucleobase deprotection and recrystallisation yielded $\mathbf{1 0 1}$ as a 35:1 mixture in favour of the desired $\beta$-anomer. Ester cleavage then leads to gemcitabine.

\subsubsection{Tosylate leaving group}

The crystalline lactone $\mathbf{4 5}$ (see 2.2.3.3, Scheme 10) was reduced with lithium tri-t-butoxyaluminium hydride, ${ }^{26}$ and then directly converted to the crystalline anomeric mixture of tosylate $\mathbf{1 0 2}$ (Scheme 26). Interestingly, the analogous mesylate was found to be an oil. It is reported that the base employed in the tosylation has an effect upon the anomeric ratio of the resultant tosylate; however, no anomeric ratios are given. In any case, a 1:1 mixture of anomers 103 was obtained regardless of the anomeric composition of the tosylate, which agrees with Chou's results on their mesylate, ${ }^{21}$ in an impressive, almost quantitative yield. The fact that a pure mixture of solid tosylate anomers could be obtained allowed the use of just 1 equiv of the expensive TMSOTf promotor for the nucleobase introduction.

The unstable 103 was not isolated, but hydrolysed to give the $\mathrm{N}$ acetyl protected cytidine derivative 104. The $\alpha$-anomer was found to precipitate from the reaction mixture, allowing its removal by filtration, giving the desired $\beta$-anomer. This material was subsequently deprotected with $\mathrm{NH}_{3}$ and converted to the gemcitabine $\mathrm{HCl}$ salt. Recrystallisation from acetone/water furnished the gemcitabine $\mathrm{HCl}$ salt with a purity of $99.8 \%$.

A variation on this process was published by Zelikovitch et al., ${ }^{45}$ where employing a different solvent combination after nucleobase introduction caused precipitation of both anomers (Scheme 27). In this way a mixture of $\mathbf{1 0 4}$ was obtained (99\% yield) containing 73\% $\beta$-anomer and $12 \% \alpha$-anomer. After deprotection of the cinnamoyl groups, recrystallisation from acetone/water, provided the $\beta$ anomer of gemcitabine. $\mathrm{HCl}$ in $99.6 \%$ purity.

\subsubsection{Ester leaving groups}

Born et al. reported the use of acetate as a leaving group for the uracil introduction (Scheme 28). ${ }^{46}$ Treatment of acetate $\mathbf{1 0 6}$ with

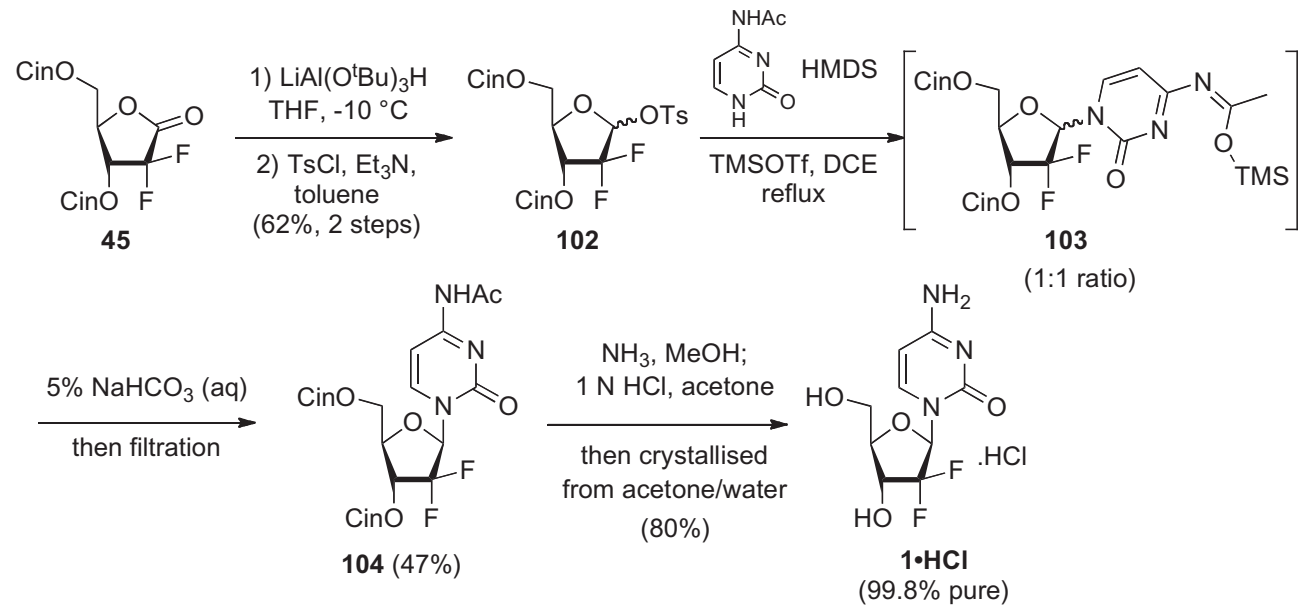

Scheme 26. Nucleobase addition by tosylate displacement (1). ${ }^{26}$ 


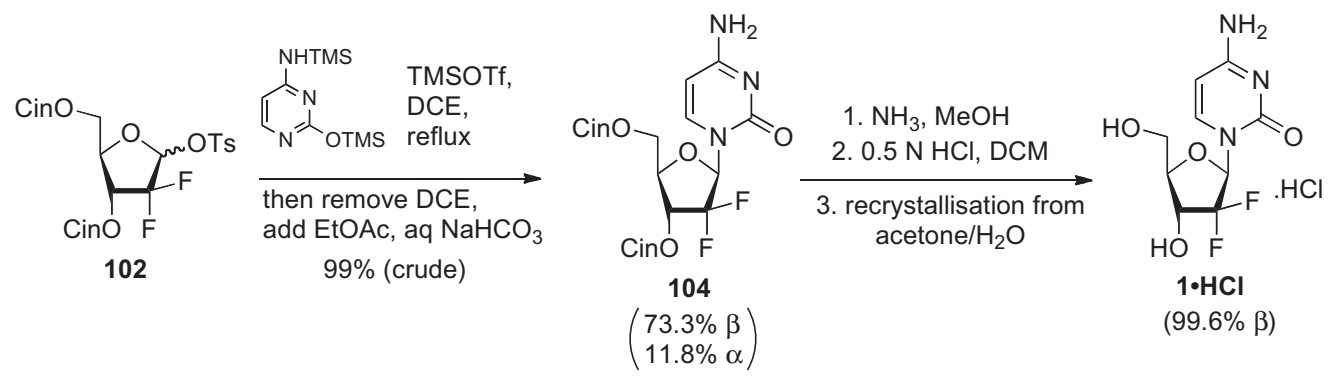

Scheme 27. Nucleobase addition by tosylate displacement (2). ${ }^{45}$

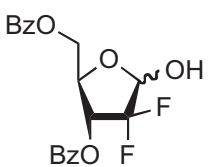

34

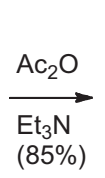

$\sum_{106}^{\mathrm{BzO}} \mathrm{OAc}$

$(1.19: 1 \beta / \alpha)$

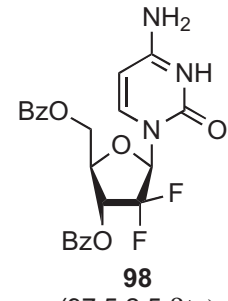

$(97.5: 2.5 \beta / \alpha)$
Scheme 28. Nucleobase addition via an acetate leaving group. ${ }^{46}$

bis-silyluracil in the presence of $\mathrm{SnCl}_{4}$ yielded the protected nucleoside 98 as a 5:1 $\beta / \alpha$ anomeric mixture. This anomeric ratio could be further enhanced by trituration to a $95 \% \mathrm{de}$.

In a similar vein, Chen reported a nucleobase introduction from the tri-O-benzoate 67 (Scheme 29), ${ }^{32}$ leading to 98 in excellent yield and anomeric ratio after a crystallisation procedure.

\subsubsection{Bromide leaving group}

Chang reported that nucleobase introduction was possible in excellent yield starting from the corresponding $\alpha$-difluororibosyl bromide donor 108 (Scheme 30). ${ }^{24 a, 46}$ While the optimisation of the nucleobase introduction reaction led to an excellent anomeric ratio, revealing some interesting mechanistic aspects of the reaction in the process, anomerically pure gemcitabine was ultimately obtained by a crystallisation process involving a hemihydrate form.

A key feature of the process was the synthesis of the donor in anomerically pure form. The required bromide was obtained by first phosphorylating the crude lactol $\mathbf{4 4}$ to give the diphenylphosphate $\mathbf{1 0 7}$ as a 1:10.8 $\alpha / \beta$ mixture. Recrystallisation from IPA/water enhanced this ratio to $>98: 2 \alpha / \beta$. However, this anomeric enhancement was ultimately unnecessary as the anomeric purity of the phosphate $\mathbf{1 0 7}$ had no effect upon the anomeric selectivity of the subsequent bromination step. The bromide $\mathbf{1 0 8}$ was obtained as a 10.8:1 $\alpha / \beta$ mixture, which again could be enhanced by recrystallisation from IPA to $>99.7: 0.3 \alpha / \beta$. Interestingly, the 4-phenyl benzoate group, employed to assist the separation of diastereomers obtained in the Reformatsky reaction as described above, proved also essential for the crystallisation process of $\mathbf{1 0 8}$, as the corresponding dibenzoate is an oil.

Initial studies found protected nucleoside $\mathbf{1 0 1}$ to be formed as a 1:1 anomeric mixture, when bromide $\mathbf{1 0 8}$ was reacted with disilylcytosine. This total lack of anomeric selectivity was presumed to be due to anomerisation of the bromide 108 , either via an $S_{N} 1$ process, or via an $\mathrm{S}_{\mathrm{N}} 2$ reaction promoted by TMSBr formed in the reaction mixture. A control experiment involving treatment of $\boldsymbol{\alpha - 1 0 8}$ with $\mathrm{TMSBr}$ indeed led to the formation of a small amount of $\beta$-anomer. Even if only a small amount of $\beta$-anomer was observed, its greater reactivity would explain the formation of a large amount of $\alpha-101$. Indeed when the TMSBr was removed from the reaction mixture via continuous distillation, using heptane as a co-solvent, the anomeric selectivity increased to 5.5:1 $\beta / \alpha$, a significant improvement. In addition, a non-polar solvent system was also utilised to minimise the $S_{N} 1$ process. Deprotection of the 5.5:1 $\beta / \alpha$ mixture of 101 with $\mathrm{NH}_{3}$, and recrystallisation from water yielded gemcitabine in greater than $99.8 \%$ anomeric purity, either as gemcitabine hemihydrate-if the mixture was stirred during the crystallisation-or as gemcitabine dihydrate-if it was not stirred.

In their patent application of the same synthesis it was also disclosed that the a small amount of additional silyl donor $(\mathrm{N}, \mathrm{O}$ bis(trimethylsilyl)acetamide, $1 \% \mathrm{v} / \mathrm{v}$ ) to the nucleobase addition reaction further enhances the anomeric ratio of $\mathbf{1 0 1}$ to $14: 1 \beta / \alpha$, however yield was not given.

\subsubsection{Iodide leaving group}

Chou et al. demonstrated the possibility for nucleobase introduction by employing iodide as the leaving group. In their patent,

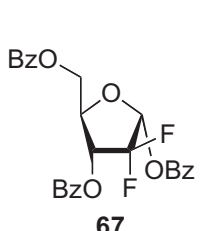

67

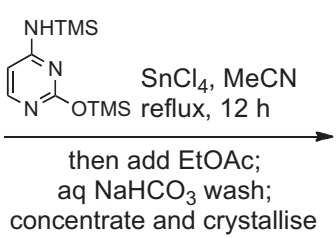

$(83 \%)$

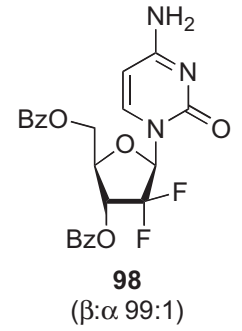<smiles>Nc1ccn(C2(O)COCC(O)C2O)c(=O)n1</smiles>

1 


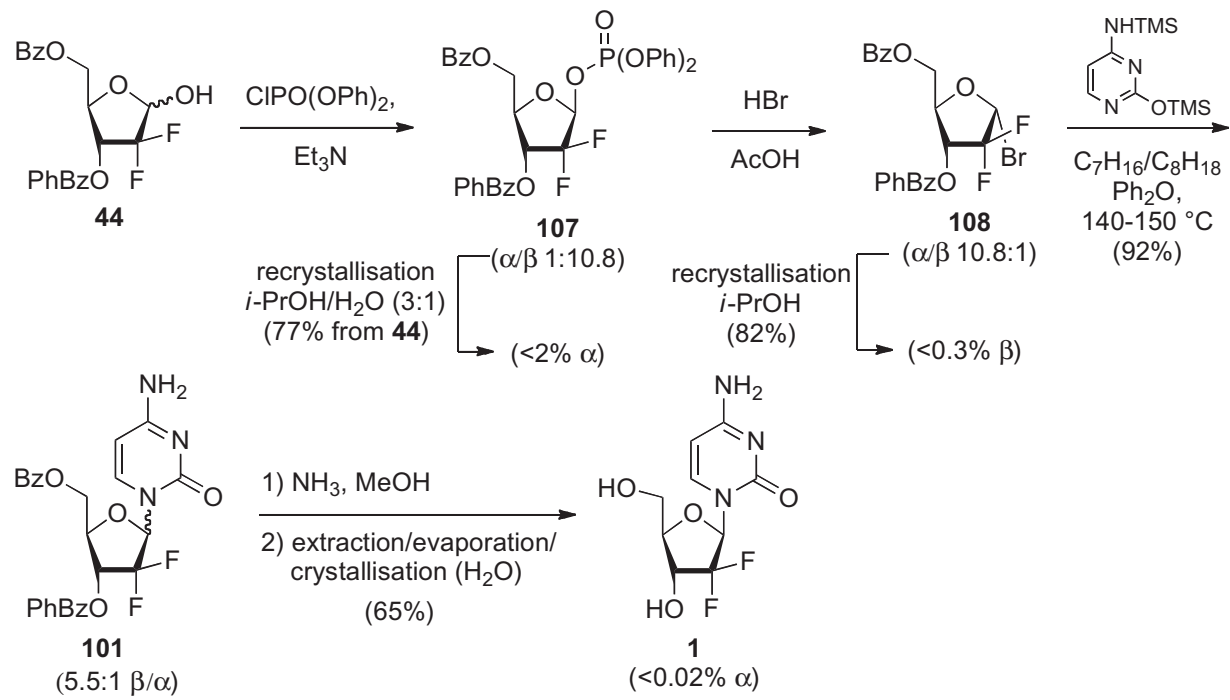

Scheme 30. Nucleobase addition via bromide displacement. ${ }^{24 a, 47}$
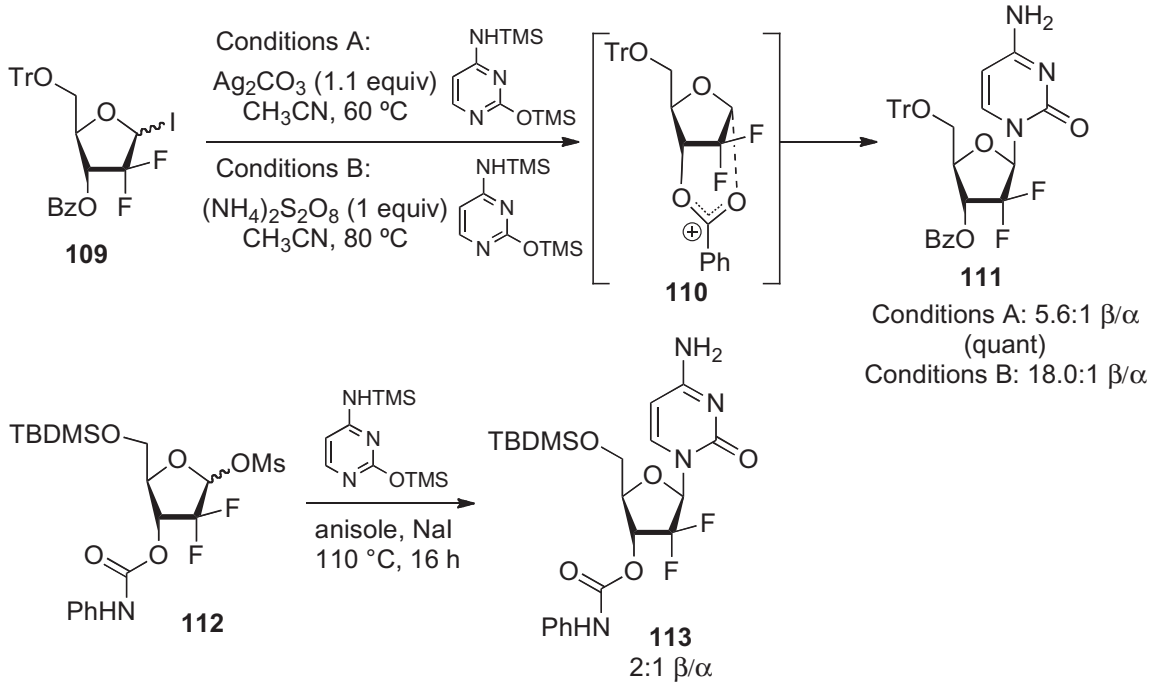

Scheme 31. Nucleobase addition with iodide leaving group. ${ }^{49,50,13}$

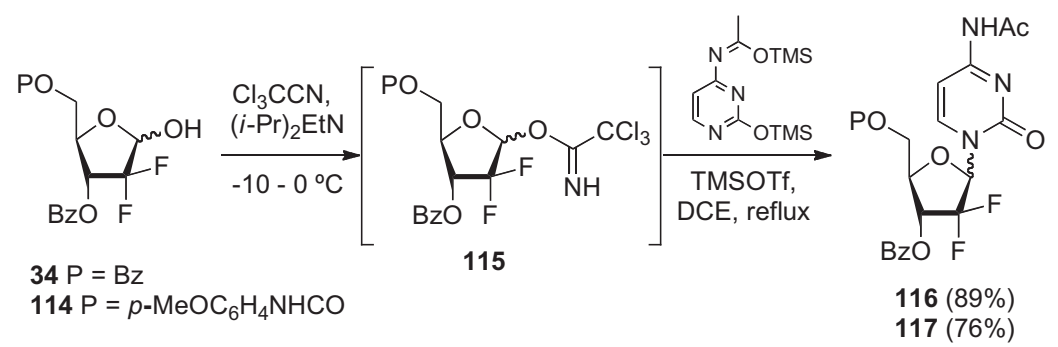

Scheme 32. Nucleobase addition-trichloroacetimidate leaving group. ${ }^{51,52}$

reaction of the potassium salt of $N$-pivaloylcytosine with the $\alpha$-enriched 3,5-di-O-benzoyl protected donor proceeded with full conversion, and in a modest $1.13: 1 \beta / \alpha$ ratio. ${ }^{48}$ Chu et al. managed to increase the anomeric ratio by employing $>1$ equiv of silver carbonate as additive (Scheme 31, conditions A) ${ }^{49}$ The iodide $\mathbf{1 0 9}$ is synthesised from the corresponding lactol via the mesylate, or via direct iodination using $\mathrm{I}_{2}$ and $\mathrm{PPh}_{3}$ in dichloromethane. Though the anomeric ratio of the donor is not specified, the 5.6:1 $\beta / \alpha$-selectivity for the nucleobase introduction is explained via invoking an $\mathrm{S}_{\mathrm{N}} 1$ type mechanism whereby the formation of the [destabilised] oxonium intermediate is facilitated by $\mathrm{Ag}(\mathrm{I})$, with stabilisation provided by neighboring group participation of the 3-O-benzoate 


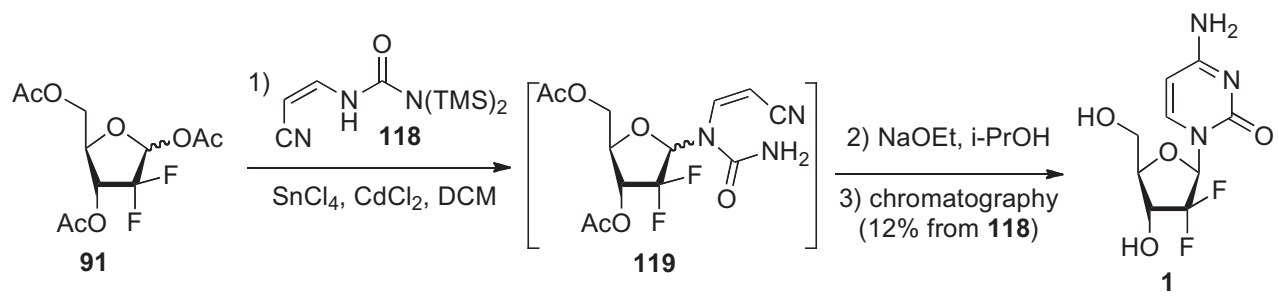

Scheme 33. Linear nucleobase synthesis. ${ }^{37}$
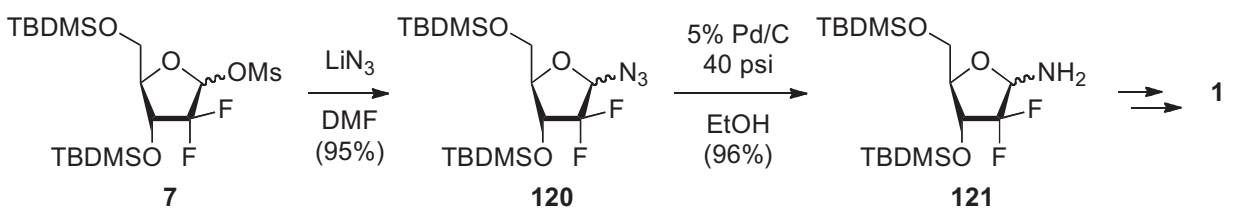

Scheme 34. Synthesis of primary aminoglycoside 121.

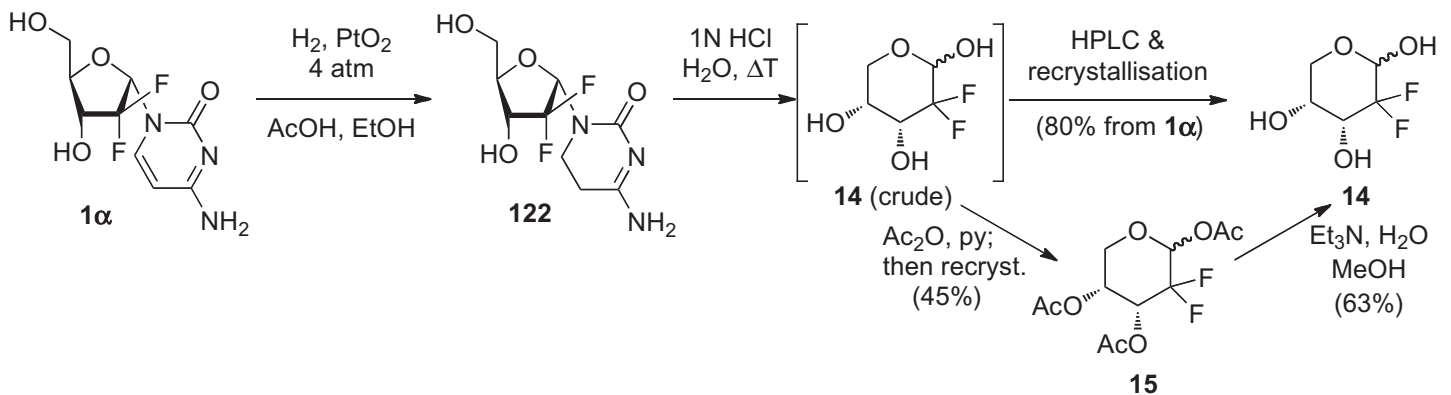

Scheme 35. Recycling of 2-deoxy-2,2-difluororibose from the $\alpha$-anomer of gemcitabine. ${ }^{38}$

group (110). Hence the bottom face of the ribose ring is blocked for nucleophilic attack thereby enhancing the formation of the $\beta$-anomer. Remarkably, this reaction is reported to proceed in quantitative yield.

Chien et al. used a different activating system to achieve the formation of $\mathbf{1 1 1}$ (Scheme 31, conditions B).$^{50}$ By using 1 equiv of an oxidant, released iodide is oxidised to $\mathrm{I}_{2}$, which is thought to assist oxonium ion formation through stabilisation of the iodide leaving group as $\mathrm{I}_{3}^{-}$. Though no yield is given, very high anomeric ratios of $\mathbf{1 1 1}$ were obtained. This method does not work well starting from the corresponding bromide or chloride donor.

Nucleobase introduction starting from the mesylate $\mathbf{1 1 2}$ in the presence of $\mathrm{NaI}$ also gives an increased $\beta$-ratio. ${ }^{13}$ Presumably this reaction proceeds via the corresponding anomeric iodide, and may involve neighboring group participation as well.

\subsubsection{Trichloroacetimidate leaving group}

Maikap et al. ${ }^{51}$ and Vishnujant et al. ${ }^{52}$ have both reported the use of trichloroacetimidate as a leaving group in the nucleobase introduction step (Scheme 32). The trichloroacetimidate donor 115 was prepared from the lactol, and nucleobase introduction was reported to proceed in good yields, but no anomeric ratio was specified.

\subsection{Nucleobase introduction-linear nucleobase synthesis}

The nucleobase can also be introduced via a linear sequence from the corresponding glycosyl amine derivative. Despite the difficulties in introducing the nucleobase via a convergent pathway (see above), very few reports involving this alternative are available.

\subsubsection{From the anomeric acetate}

Glycosylation of peracetylated 2-deoxy-2,2-difluororibose 91 (Scheme 33) with $N, N$-bistrimethylsilyl-((Z)-2-cyanovinylurea) 118, synthesised from the corresponding cyanovinylurea, under Lewis-acid activation led to the nucleoside precursor $119 .{ }^{37}$ Basemediated cyclisation resulted in cytosine ring formation with concomitant protecting group removal. Though no anomeric ratio was provided, silica gel based chromatography led to gemcitabine $\mathbf{1}$ in $12 \%$ overall yield.

\subsubsection{Synthesis of the anomeric amine precursor}

An alternative way for the synthesis of pyrimidine nucleosides employs anomeric aminoglycoside derivatives as starting material. Hertel et al. disclosed the synthesis of the primary aminoglycoside 121 (Scheme 34) as suitable precursor for a linear gemcitabine synthesis. ${ }^{53}$ Nucleophilic substitution of mesylate 7 by azide led to 120 in excellent yield. The synthesis of the analogous benzyl protected aminoglycoside was also reported. It was found that the azide introduction proceeded with inversion of configuration: when the $\alpha$-mesylate was used, the $\beta$-azide was isolated in $76 \%$ yield; starting from the $\beta$-mesylate, the $\alpha$-azide was isolated in 73\% yield. Azide reduction to the amine $\mathbf{1 2 1}$ then proceeded in near quantitative yield, and a 1:1 anomeric ratio was obtained regardless of the configuration of the starting azide. 


\subsection{Recycling of the $\alpha$-anomer}

Given no fully selective nucleobase introduction method has been developed so far, all current syntheses yield a quantity of undesired $\alpha$-anomer of gemcitabine, and a number of methods have been developed to convert this byproduct into gemcitabine, or at least to recover the valuable difluororibose.

Britton showed that pure $\alpha$-anomer could be isomerised to the $\beta$-anomer by treatment with a hydroxide base in an anhydrous alcohol solvent, up to a ratio of 35:65 $\alpha: \beta .^{54}$

Nagarajan, from the Lilly labs, developed a process to recover 2-deoxy-2,2-difluororibose from the unwanted $\alpha$-anomer of gemcitabine (Scheme 35). ${ }^{38}$ In order to remove the nucleobase group by hydrolysis at the anomeric centre, a process disfavoured by the presence of the fluorination at the $\mathrm{C} 2$ position, the pyrimidine ring was first partially hydrogenated using a $\mathrm{PtO}_{2}$ catalyst at medium hydrogen gas pressure to give $\mathbf{1 2 2}$. This then enabled acid-catalysed hydrolysis using strong mineral acid at high temperature (steam bath), yielding the 2-deoxy-2,2-difluororibose sugar 14. Purification is necessary, and this can be achieved by column chromatography and recrystallisation ( $80 \%$ yield from $1 \alpha$ ), or alternatively, by acetylating the crude reaction mixture to give the triacetate $\mathbf{1 5}$, followed by deprotection and recrystallisation. However, this second procedure leads to difluororibose in only $28 \%$ overall yield.

\subsection{Conclusion}

This review illustrates how the quest for an efficient, scalable synthesis of gemcitabine has spurned enormous synthetic efforts towards 2-deoxy-2,2-difluororibose and its nucleobase introduction. It provides a nice overview of the different strategies for $\mathrm{CF}_{2}$-introduction in a sugar moiety: a building block approach, electrophilic $\alpha$-fluorination of esters (lactones) and nucleophilic fluorination of ketones. Finally, it is shown how difluorination at a sugar 2-position necessitates less conventional methods for nucleobase introduction. The review also gives a taste of how different protecting groups can be used to find conditions to separate diastereomers by crystallisation. Despite all the efforts, there is still scope for increasing selectivities and yields, and with the recent expiration of the Lilly gemcitabine patent, it is certain that further research to that effect will continue.

\section{Acknowledgement}

KB thanks Dextra Laboratories and the EPSRC for a KTS Secondment (GR/T18882/01).

\section{References}

1. Reviews about fluorinated nucleosides: (a) Wojtowicz-Rajchel, H. J. Fluorine Chem. 2012, 143, 11-48; (b) Qiu, X.-L.; Xu, X.-H.; Qing, F.-L. Tetrahedron 2010 66, 789-843; (c) Liu, P.; Sharon, A.; Chu, C. K. J. Fluorine Chem. 2008, 129, $743-$ 766; (d) Watts, J. K.; Damha, M. J. Can. J. Chem. 2008, 86, 641-656; (e) Meng W.-D.; Qing, F.-L. Curr. Top. Med. Chem. 2006, 6, 1499-1528; (f) Pankiewicz, K. W. Carbohydr. Res. 2000, 327, 87-105.

2. http://thomsonreuters.com/cortellis-for-competitive-intelligence/.

3. Martindale 2011. The complete Drug Reference; Sweetman, S. C., Ed.; Royal Pharmaceutical Society: London, 2011. 37th ed.

4. (a) Silvestris, N.: Cinieri, S.; La Torre, I.; Pezzella, G.; Numico, G.; Orlando, L.; Lorusso, V. The Breast 2008, 17, 220-226; (b) Vulfovich, M.; Rocha-Lima, C. Expert Rev. Anticancer Ther. 2008, 8, 993-1002; (c) Lorusso, D.; Di Stefano, A.; Fanfani, F.; Scambia, G. Ann. Oncol. 2006, 17, V188-V194.
5. Recent example: Maiti, S.; Park, N.; Han, J. H.; Jeon, H. M.; Lee, J. H.; Bhuniya, S.; Kang, C.; Kim, J. S. J. Am. Chem. Soc. 2013, 135, 4567-4572.

6. Hertel, L. W.; Kroin, J. S.; Misner, J. W.; Tustin, J. M. J. Org. Chem. 1988, 53, 2406 2409.

7. Baer, E.; Fischer, H. O. L. J. Biol. Chem. 1939, 128, 463-473.

8. Hallinan, E. A.; Fried, J. Tetrahedron Lett. 1984, 25, 2301-2302.

9. Reviews: (a) Choppin, S.; Ferreiro-Medeiros, L.; Barbarotto, M.; Colobert, F. Chem. Soc. Rev. 2013, 42, 937-949; (b) Ocampo, R.; Dolbier, W. R., Jr. Tetrahedron 2004, 60, 9325-9374; (c) Furstner, A. Synthesis 1989, 571-590.

10. Kitagawa, O.; Taguchi, T.; Kobayashi, Y. Tetrahedron Lett. 1988, 29, 1803-1806.

11. (a) Kotra, L. P.; Xiang, Y.; Newton, M. G.; Schinazi, R. F.; Cheng, Y.-C.; Chu, C. K.J. Med. Chem. 1997, 40, 3635-3644; (b) Xiang, Y.; Kotra, L. P.; Chu, C. K.; Schinazi, R. F. Bioorg. Med. Chem. Lett. 1995, 5, 743-748.

12. Hubschwerlen, C. Synthesis 1986, 962-964.

13. Lin, K.-C.; Li, W.; Lin, C.; Wein, Y.; Lai, Y.; Kao, K.-H.; Lu, M.-Y. W0119347, 2006.

14. a) Noe, C. R.; Jasic, M.; Kollmann, H.; Saadat, K. WO009147, 2007.; b) Noe, C. R.; Jasic, M.; Kollmann, H.; Saadat, K. USO249119, 2008. Note: no stereochemistry was indicated in the patent and yields were calculated from crude masses provided.

15. Hanzawa, Y.; Inazawa, K.; Kon, A.; Aoki, H.; Kobayashi, Y. Tetrahedron Lett. 1987, 28, 659-662.

16. Wirth, D. D. EP0727432, 1996.

17. Weigel, J. A. J. Org. Chem. 1997, 62, 6108-6109.

18. Matsumura, Y.; Fujii, H.; Nakayama, T.; Morizawa, Y.; Yasuda, A. J. Fluorine Chem. 1992, 57, 203-207.

19. Shen, X.; Liao, L.; Lin, F.; He, X.; Yang, J.; Zhan, H. CN101469010, 2009. Note: the D-glyceraldehyde acetonide starting material was shown with the wrong absolute configuration, and the addition product shown as the syndiastereomer. See also INMU00450, 2008.

20. Zhang, Y.; Sammakia, T. J. Org. Chem. 2006, 71, 6262-6265.

21. Chou, T. S.; Heath, P. C.; Patterson, L. E.; Poteet, L. M.; Lakin, R. E.; Hunt, A. H. Synthesis 1992, 565-570.

22. Kim, M.-S.; Kim, Y.-J.; Choi, J.-H.; Lim, H.-G.; Cha, D.-W. US0281301, 2009

23. Example: Potluri, R. B.; Venkata Subramanian, H.; Betini, R.; Gunturu, S. W0095359, 2006.

24. (a) Chang Y-K. Lee, J; Park, G.-S.; Lee, M. Park, C. H.; Kim, H. K.; Lee, G.; Lee, B.-Y.; Baek, J. Y.; Kim, K. S. Tetrahedron 2010, 66, 5687-5691; (b) Lee, J.; Park, G.-S.; Lee, M.; Kim, C.-K.; Lee, J.-C.; Chang, Y.-K.; Lee, G.-S. WO009353, 2006.

25. a) Xu, Y.; Yang, H.; Hou, W. US0179314, 2010.

26. (a) Jiang, X.; Li, J.; Zhang, R.; Zhu, Y.; Shen, J. Org. Process Res. Dev. 2008, 12 888-891; (b) See also Shen, J.; Li, Y.; Kaspi, J. WO027564, 2007.

27. Naddaka, V.; Klopfer, E.; Saeed, S.; Montvilisky, D.; Arad, O.; Kaspi, J. WO070804, 2007.

28. Park, S.-J.; Oh, C.-R.; Kim, Y.-D. WO117955, 2008.

29. Cen, Y.; Sauve, A. A. J. Org. Chem. 2009, 74, 5779-5789.

30. Cen, Y.; Sauve, A. A. Nucleosides, Nucleotides Nucleic Acids 2010, 29, 113-122.

31. McAtee, J. J.: Schinazi, R. F. Liotta, D. C. J. Org. Chem. 1998, 63, 2161-2167.

32. Gong, C. CN101628927A, 2010.

33. (a) Brodfuehrer, P. R.; Sapino, C., Jr.; Howell, H. G. J. Org. Chem. 1985, 50, $2597-$ 2598: (b) Howell, H. G.; Brodfuehrer, P. R.; Brundidge, S. P.; Benigni, D. A. Sapino, C., Jr. J. Org. Chem. 1988, 53, 85-88.

34. Fernandez, R.; Matheu, M. I.; Echarri, R.; Castillon, S. Tetrahedron 1998, 54, 3523-3532.

35. Horton, D.: Weckerle, W. Carbohydr. Res. 1975, 44, 227-240.

36. El-Laghdach, A.; Echarri, R.; Matheu, M. I.; Barrena, M. I.; Castillon, S.; Garcia, J. J. Org. Chem. 1991, 56, 4556-4559.

37. Gong, C. US0003963, 2006. In the patent; 14 is depicted in the furanose form.

38. Nagarajan, R. US4954623, 1990.

39. Wirth, D. D. EP0727433, 1996.

40. Kjell, D. P. US5633367, 1997.

41. Chou, T.-S.; Poteet, L. M.; Kjell, D. P.; Grossman, C. S.; Hertel, L. W.; Holmes, R. E.; Jones, C. D.; Mabry, T. E. EP0577303, 1994

42. Chou, T. S. US5401838, 1995

43. Kjell, D. P. US5426183, 1995.

44. Wildfeuer, M. E. US5521294, 1996.

45. Zelikovitch, L.; Friedman, O.; Fizitzky, T.; Manascu, J. US0262215, 2008.

46. Born, A.-R.; Martin, P.; Spielvogel, D.; Villa, M. WO063105, 2006.

47. Lee, J.; Park, G. S.; Lee, M.; Bang, H.-J.; Lee, J. C.; Kim, C. K.; Choi, C.-J.; Kim, H. K.; Lee, H. C.; Chang, Y.-K.; Lee, G. S. US0249818, 2007.

48. Chou, T.-S.; Grossman, C. S.; Hertel, L. W.; Holmes, R. E.; Jones, C. D.; Mabry, T. E. EP0577304, 1994.

49. Chu, C.-Y.; Lee, W.-D.; Li, W.; Hwang, C. K. US0124797, 2009.

50. Chien, C.; Chien, P.-S.; Hwang, C.-K. EP2508528, 2012.

51. Maikap, G. C.; Bhatt, D.; Panda, B. K. W0092808, 2006.

52. Vishnukant, B.; Purohit, P.; Paparao, K.; Veereshappa, V. WO026222, 2008

53. Hertel, L. W.; Jones, C. D.; Kroin, J. S.; Mabry, T. E. US5594155, 1997.

54. Britton, T. C.; LeTourneau, M. E. US5420266, 1995 